Article

\title{
On the Sharp Gårding Inequality for Operators with Polynomially Bounded and Gevrey Regular Symbols
}

\author{
Alexandre Arias Junior ${ }^{1}$ (D) and Marco Cappiello ${ }^{2, *}$ \\ 1 Department of Mathematics, Federal University of Paraná, Curitiba 81531-980, Brazil; arias@ufpr.br \\ 2 Department of Mathematics, University of Turin, Via Carlo Alberto 10, 10123 Turin, Italy \\ * Correspondence: marco.cappiello@unito.it
}

Received: 29 September 2020; Accepted: 29 October 2020; Published: 3 November 2020

\begin{abstract}
In this paper, we analyze the Friedrichs part of an operator with polynomially bounded symbol. Namely, we derive a precise expression of its asymptotic expansion. In the case of symbols satisfying Gevrey estimates, we also estimate precisely the regularity of the terms in the asymptotic expansion. These results allow new and refined applications of the sharp Gårding inequality in the study of the Cauchy problem for $p$-evolution equations.
\end{abstract}

Keywords: pseudodifferential operators; Gevrey regularity; sharp Gårding inequality; $p$-evolution equations

MSC: 35S05; 46F05; 35S10

\section{Introduction}

The sharp Gårding inequality for a pseudodifferential operator was first proved by Hörmander [1] and by Lax and Nirenberg [2] for symbols in the Kohn-Nirenberg class $S^{m}\left(\mathbb{R}^{2 n}\right)$, namely satisfying the following estimates

$$
\left|\partial_{\xi}^{\alpha} \partial_{x}^{\beta} p(x, \xi)\right| \leq C_{\alpha \beta}\langle\xi\rangle^{m-|\alpha|}, \quad \alpha, \beta \in \mathbb{N}_{0}^{n},
$$

for some positive constant $C_{\alpha \beta}$, where $\langle\xi\rangle:=\sqrt{1+|\xi|^{2}}$ for every $\xi \in \mathbb{R}^{n}$ and $\mathbb{N}_{0}^{n}$ stands for the set of all multi-indices of length $n$. In its original form, this result states that, if $p \in S^{m}\left(\mathbb{R}^{2 n}\right)$, for some $m \in \mathbb{R}$, is such that $\operatorname{Re} p(x, \xi) \geq 0$, then the corresponding operator $p(x, D)$ satisfies the following estimate

$$
\operatorname{Re}(p(x, D) u, u)_{L^{2}} \geq-C\|u\|_{\frac{m-1}{2}}^{2}, \quad u \in \mathscr{S}\left(\mathbb{R}^{n}\right)
$$

for some $C \in \mathbb{R}$, where $\|\cdot\|_{\frac{m-1}{2}}$ denotes the standard norm in the Sobolev space $H^{\frac{m-1}{2}}\left(\mathbb{R}^{n}\right)$. Later on, several different proofs and extensions of this result have been provided by many authors (cf. [3-6]). In particular, the inequality has been extended to symbols defined in terms of a general metric (cf. [4], Theorem 18.6.7) and to matrix valued pseudo-differential operators (cf. [4], Lemma 18.6.13, and [5], Theorem 4.4 page 134). In all the proofs of the sharp Garding inequality, the operator $p(x, D)$ is decomposed as the sum of a positive definite part and a remainder term providing the inequality (1). In the approach proposed in [5], this positive definite part $p_{F}$ is called Friedrichs part and satisfies the following conditions:

(i) $\quad\left(p_{F} u, v\right)_{L^{2}}=\left(u, p_{F} v\right)_{L^{2}}$ if $p(x, \xi)$ is real;

(ii) $\left(p_{F} u, u\right)_{L^{2}} \geq 0$ if $p(x, \xi) \geq 0$; and

(iii) $\operatorname{Re}\left(p_{F} u, u\right)_{L^{2}} \geq 0$ if $\operatorname{Re} p(x, \xi) \geq 0$.

Although the results in [4] are extremely general and sharp, in some applications, more detailed information on the remainder term is needed. In particular, it is important to state not only the order 
but also the asymptotic expansion of $p-p_{F}$. This is needed in particular in the analysis of the so called $p$-evolution equations, namely equations of the form

$$
D_{t} u+a_{p}(t) D_{x}^{p}+\sum_{j=0}^{p-1} a_{j}(t, x) D_{x}^{j} u=f(t, x), \quad t \in[0, T],
$$

where $p$ is a positive integer and $a_{p}(t)$ is a real valued function (cf. [7,8]). This large class of equations includes for instance strictly hyperbolic equations $(p=1)$ and Schrödinger-type equations $(p=2)$. The classical approach to study the Cauchy problem for these equations is based on a reduction to an auxiliary problem via a suitable change of variable and on a repeated application of sharp Gårding inequality which needs at every step to understand the precise form of all the remainder terms (cf. [9]). When the coefficients $a_{j}(t, x)$ are uniformly bounded with respect to $x$, this is possible using Theorem 4.2 in [5], where the asymptotic expansion of $p_{F}-p$ is given in the frame of classical Kohn-Nirenberg classes. In this way, under suitable assumptions on the behavior of the coefficients $a_{j}(t, x), j=0, \ldots, p-1$, for $|x| \rightarrow \infty$, well posedness with loss of derivatives has been proved in $H^{\infty}=\cap_{m \in \mathbb{R}} H^{m}$ (see [9]).

In fact, for equations of the form (2), the loss of derivatives can be avoided by choosing the data of the Cauchy problem with a certain decay at infinity (cf. [10]). This motivated us to study the initial value problem for (2) in a weighted functional setting admitting also polynomially bounded coefficients, which cannot be treated in the theory of standard Kohn-Nirenberg classes but are included in the so-called SG classes (see the definition below). For this purpose we need a variant of [5] (Theorem 4.2) for SG operators with a precise information on the asymptotic expansion of $p-p_{F}$.

Another challenging issue is to study Equation (2) on Gelfand-Shilov spaces of type $\mathcal{S}$ (cf. [11]). A first step in this direction has been done in the case $p=2$, that is for Schrödinger-type equations (see [12]), and for $p=3$ (see [13]). In both cases, it is sufficient to apply the sharp Gårding inequality only once. To treat $p$-evolution equations for $p>3$, however, we need to apply the iterative procedure described above. In addition, a precise estimate of the Gevrey regularity of the terms in the asymptotic expansion of $p-p_{F}$ is also needed.

In this paper, we provide appropriate tools for both the aforementioned issues. This is achieved by defining in a suitable way the Friedrichs part of our operators and by studying in detail its asymptotic expansion and its regularity. With this purpose, we prove two separate results for the following classes of symbols. Fixing $m=\left(m_{1}, m_{2}\right) \in \mathbb{R}^{2}$, we denote by $\mathbf{S G}^{m}\left(\mathbb{R}^{2 n}\right)$ the space of all functions $p \in C^{\infty}\left(\mathbb{R}^{2 n}\right)$ satisfying for any $\alpha, \beta \in \mathbb{N}_{0}^{n}$ the following condition

$$
\left|\partial_{\xi}^{\alpha} \partial_{x}^{\beta} p(x, \xi)\right| \leq C_{\alpha \beta}\langle\xi\rangle^{m_{1}-|\alpha|}\langle x\rangle^{m_{2}-|\beta|}, \quad x, \xi \in \mathbb{R}^{n},
$$

for some positive constant $C_{\alpha, \beta}$. These symbols have been treated by a large number of authors along the years (see [14-21]). We are moreover interested in the subclass of $\mathbf{S G} \mathbf{G}^{m}\left(\mathbb{R}^{2 n}\right)$ given by the $\mathbf{S G}$ symbols possessing a Gevrey-type regularity. Namely, for $\mu, v \geq 1$, we say that a symbol $p(x, \xi)$ belongs to the class $\mathbf{S G}_{(\mu, v)}^{m}\left(\mathbb{R}^{2 n}\right)$ if there are constants $C, C_{1}>0$ satisfying

$$
\left|\partial_{\xi}^{\alpha} \partial_{x}^{\beta} p(x, \xi)\right| \leq C_{1} C^{|\alpha|+|\beta|} \alpha !^{\mu} \beta !^{\nu}\langle\xi\rangle^{m_{1}-|\alpha|}\langle x\rangle^{m_{2}-|\beta|},
$$

for every $\alpha, \beta \in \mathbb{N}_{0}^{n}, x, \xi \in \mathbb{R}^{n}$.

This work is organized as follows. In Section 2, we recall some results concerning SG pseudodifferential operators. In Section 3, we discuss the concepts of oscillatory integrals and double symbols, which are fundamental tools in the present work. Finally, in Section 4, we study the Friedrichs part of symbols belonging to the classes $\mathbf{S G}^{m}$ and $\mathbf{S G}_{(\mu, v)}^{m}$ and we prove the main results of this paper, namely Theorems 4 and 6 . 


\section{SG Pseudodifferential Operators}

In this section, we recall some basic facts about SG pseudodifferential operators which are used in the sequel. Although for our applications we are interested to prove the main results for the classes defined by inequalities (3) and (4), in order to prove them, we need to consider more general classes of symbols which are defined as follows.

Definition 1. Given $m=\left(m_{1}, m_{2}\right) \in \mathbb{R}^{2}, \rho=\left(\rho_{1}, \rho_{2}\right) \in(0,1]^{2}, \delta=\left(\delta_{1}, \delta_{2}\right) \in[0,1)^{2}$, with $\delta_{j}<\rho_{j}, j=1,2$, we denote by $S G_{\rho, \delta}^{m}$ the space of all functions $p \in C^{\infty}\left(\mathbb{R}^{2 n}\right)$ such that

$$
\sup _{(x, \xi) \in \mathbb{R}^{2 n}}\left|\partial_{\xi}^{\alpha} \partial_{x}^{\beta} p(x, \xi)\right|\langle\xi\rangle^{-m_{1}+\rho_{1}|\alpha|-\delta_{1}|\beta|}\langle x\rangle^{-m_{2}+\rho_{2}|\beta|-\delta_{2}|\alpha|}<\infty .
$$

We recall that $\mathbf{S G}_{\rho, \delta}^{m}$ is a Fréchet space endowed with the seminorms

$$
|p|_{\ell}:=\sup _{\substack{(x, \xi) \in \mathbb{R}^{2 n} \\|\alpha+\beta| \leq \ell}}\left|\partial_{\xi}^{\alpha} \partial_{x}^{\beta} p(x, \xi)\right|\langle\xi\rangle^{-m_{1}+\rho_{1}|\alpha|-\delta_{1}|\beta|}\langle x\rangle^{-m_{2}+\rho_{2}|\beta|-\delta_{2}|\alpha|},
$$

for $\ell \in \mathbb{N}_{0}$. The class $\mathbf{S G}_{\rho, \delta}^{m}$ is included in the general theory by Hörmander [4]. A specific calculus for this class can be found in [22]. Pseudodifferential operators with symbols in $\mathbf{S G}_{\rho, \delta}^{m}$ are linear and continuous from $\mathscr{S}\left(\mathbb{R}^{n}\right)$ to $\mathscr{S}\left(\mathbb{R}^{n}\right)$ and extend to linear and continuous maps from $\mathscr{S}^{\prime}\left(\mathbb{R}^{n}\right)$ to $\mathscr{S}^{\prime}\left(\mathbb{R}^{n}\right)$. Moreover, denoting by $H^{s}\left(\mathbb{R}^{n}\right)$ with $s=\left(s_{1}, s_{2}\right) \in \mathbb{R}^{2}$ the weighted Sobolev space

$$
H^{s}\left(\mathbb{R}^{n}\right):=\left\{u \in \mathscr{S}^{\prime}\left(\mathbb{R}^{n}\right):\langle x\rangle^{s_{2}}\left\langle D_{x}\right\rangle^{s_{1}} u \in L^{2}\left(\mathbb{R}^{n}\right)\right\},
$$

we know that an operator with symbol in $\mathbf{S G}_{\rho, \delta}^{m}$ extends to a linear and continuous map from $H^{s}\left(\mathbb{R}^{n}\right)$ to $H^{s-m}\left(\mathbb{R}^{n}\right)$ for every $s \in \mathbb{R}^{2}$.

Definition 2. Let, for $j \in \mathbb{N}_{0}, p_{j} \in S G_{\rho, \delta}^{\left(m_{1, j}, m_{2, j}\right)}$, where $m_{1, j}, m_{2, j}$ are nonincreasing sequences and $m_{1, j} \rightarrow-\infty$, $m_{2, j} \rightarrow-\infty$, when $j \rightarrow \infty$. We say that $p \in C^{\infty}\left(\mathbb{R}^{2 n}\right)$ has the asymptotic expansion

$$
p(x, \xi) \sim \sum_{j \in \mathbb{N}_{0}} p_{j}(x, \xi)
$$

if for any $N \in \mathbb{N}$ we have

$$
p(x, \xi)-\sum_{j=0}^{N-1} p_{j}(x, \xi) \in S G_{\rho, \delta}^{\left(m_{1, N}, m_{2, N}\right)} .
$$

Given $p_{j} \in \mathbf{S G}_{\rho, \delta}^{\left(m_{1, j}, m_{2, j}\right)}$ as in the previous definition, we can find $p \in \mathbf{S G}_{\rho, \delta}^{\left(m_{1,0}, m_{2,0}\right)}$ such that $p \sim \sum p_{j}$. Furthermore, if there is $q$ such that $q \sim \sum p_{j}$, then $p-q \in \mathbf{S G}^{-\infty}:=\cap_{m \in \mathbb{R}^{2}} \mathbf{S G}_{\rho, \delta}^{m}=\mathscr{S}\left(\mathbb{R}^{2 n}\right)$ (cf. [22], Theorem 2). The class $\mathbf{S G}_{\rho, \delta}^{m}$ is closed under adjoints. Namely, given $p \in \mathbf{S G}_{\rho, \delta}^{m}$ and denoting by $P^{*}$ the $L^{2}$ adjoint of $p(x, D)$, we can write $P^{*}=p^{*}(x, D)+R$, where $p^{*}$ is a symbol in $\mathbf{S G}_{\rho, \delta}^{m}$ admitting the asymptotic expansion

$$
p^{*}(x, \xi) \sim \sum_{\alpha \in \mathbb{N}_{0}^{n}} \alpha !^{-1} \partial_{\xi}^{\alpha} D_{x}^{\alpha} \overline{p(x, \xi)}
$$


and $R: \mathscr{S}^{\prime}\left(\mathbb{R}^{n}\right) \rightarrow \mathscr{S}\left(\mathbb{R}^{n}\right)$. The class $\mathbf{S G}_{\rho, \delta}^{\infty}:=\cup_{m \in \mathbb{R}^{2}} \mathbf{S G}_{\rho, \delta}^{m}$ possesses algebra properties with respect to composition. Namely, given $p \in \mathbf{S G}_{\rho, \delta}^{m}$ and $q \in \mathbf{S G}_{\rho, \delta}^{m^{\prime}}$, there exists a symbol $s \in \mathbf{S G}_{\rho, \delta}^{m+m^{\prime}}$ such that $p(x, D) q(x, D)=s(x, D)+R^{\prime}$ where $R^{\prime}$ is a smoothing operator $\mathscr{S}^{\prime}\left(\mathbb{R}^{n}\right) \rightarrow \mathscr{S}\left(\mathbb{R}^{n}\right)$. Moreover,

$$
s(x, \xi) \sim \sum_{\alpha \in \mathbb{N}_{0}^{n}} \alpha !^{-1} \partial_{\xi}^{\alpha} p(x, \xi) D_{x}^{\alpha} q(x, \xi)
$$

(cf. [22], Theorem 3).

We now consider Gevrey regular symbols.

Definition 3. Fixing $C>0$, we denote by $S G_{\rho, \delta ;(\mu, v)}^{m}\left(\mathbb{R}^{2 n} ; C\right)$ the space of all smooth functions $p(x, \xi)$ such that

$$
|p|_{C}:=\sup _{\alpha, \beta \in \mathbb{N}_{0}^{n}} C^{-|\alpha|-|\beta|} \alpha !^{-\mu} \beta !^{-v} \sup _{x, \xi \in R^{n}}\langle\xi\rangle^{-m_{1}+\rho_{1}|\alpha|-\delta_{1}|\beta|}\langle x\rangle^{-m_{2}+\rho_{2}|\beta|-\delta_{2}|\alpha|}\left|\partial_{\xi}^{\alpha} \partial_{x}^{\beta} p(x, \xi)\right|<+\infty .
$$

We set $S G_{\rho, \delta ;(\mu, v)}^{m}\left(\mathbb{R}^{2 n}\right)=\bigcup_{C>0} S G_{\rho, \delta ;(\mu, v)}^{m}\left(\mathbb{R}^{2 n} ; C\right)$.

Equipping $\mathbf{S G}_{\rho, \delta ;(\mu, v)}^{m}\left(\mathbb{R}^{2 n} ; C\right)$ with the norm $|\cdot|_{C}$ we obtain a Banach space and we can endow $\mathbf{S G}_{\rho, \delta ;(\mu, v)}^{m}\left(\mathbb{R}^{2 n}\right)$ with the topology of inductive limit of Banach spaces. A complete calculus for operators with symbols in this class can be found in [23]. Here, we recall only the main results. Since $\mathbf{S G}_{\rho, \delta ;(\mu, v)}^{m} \subset \mathbf{S G}_{\rho, \delta}^{m}$, the previous mapping properties on the Schwartz and weighted Sobolev spaces hold true for operators with symbols in $\mathrm{SG}_{\rho, \delta ;}^{m}(\mu, v)$. By the way, the most natural functional setting for these operators is given by the Gelfand-Shilov spaces of type $\mathcal{S}$. We recall that, fixing $\mu>0, v>0$, the Gelfand-Shilov space $\mathcal{S}_{v}^{\mu}\left(\mathbb{R}^{n}\right)$ is defined as the space of all functions $f \in C^{\infty}\left(\mathbb{R}^{n}\right)$ such that for some constant $C>0$

$$
\sup _{\alpha, \beta \in \mathbb{N}_{0}^{n}} C^{-|\alpha+\beta|}(\alpha !)^{-\nu}(\beta !)^{-\mu} \sup _{x \in \mathbb{R}^{n}}\left|x^{\alpha} \partial^{\beta} f(x)\right|<+\infty .
$$

For every $\mu^{\prime} \geq \mu /\left(1-\delta_{2}\right), v^{\prime} \geq v /\left(1-\delta_{1}\right)$, an operator with symbol in $\mathbf{S G}_{\rho, \delta ;(\mu, v)}^{m}$ is linear and continuous from $\mathcal{S}_{\mu^{\prime}}^{v^{\prime}}\left(\mathbb{R}^{n}\right)$ to itself and extends to a linear continuous map from the dual space $\left(\mathcal{S}_{\mu^{\prime}}^{v^{\prime}}\right)^{\prime}\left(\mathbb{R}^{n}\right)$ into itself (see [23], Theorem A.4).

The notion of asymptotic expansion for symbols in $\mathbf{S G}_{\rho, \delta ;(\mu, v)}^{m}$ can be defined in terms of formal sums (cf. [23]). Here, to obtain our results, we need to use a refined notion of formal sum introduced in [13] for the case $\rho=(1,1), \delta=(0,0)$. All the next statements can be transferred to the case of general $\rho$ and $\delta$ without changing the argument, thus we refer to [13] for the proofs.

For $t_{1}, t_{2} \geq 0$, set

$$
Q_{t_{1}, t_{2}}=\left\{(x, \xi) \in \mathbb{R}^{2 n}:\langle x\rangle<t_{1} \text { and }\langle\xi\rangle<t_{2}\right\}
$$

and $Q_{t_{1}, t_{2}}^{e}=\mathbb{R}^{2 n} \backslash Q_{t_{1}, t_{2}}$. When $t_{1}=t_{2}=t$, we simply write $Q_{t}$ and $Q_{t}^{e}$.

Definition 4. Let $\bar{\sigma}_{j}=\left(k_{j}, \ell_{j}\right)$ be a sequence such that $k_{0}=\ell_{0}=0, k_{j}, \ell_{j}$ are strictly increasing, $k_{j+N} \geq k_{j}+k_{N}, \ell_{j+N} \geq \ell_{j}+\ell_{N}$, for $j, N \in \mathbb{N}_{0}$, and $k_{j} \geq \Lambda_{1} j, \ell_{j} \geq \Lambda_{2} j$ for $j \geq 1$, for some $\Lambda_{1}, \Lambda_{2}>0$. We say that $\sum_{j \geq 0} p_{j} \in F_{\bar{\sigma}} S G_{\rho, \delta ;}^{m}(\mu, v)$ if $p_{j} \in C^{\infty}\left(\mathbb{R}^{2 n}\right)$ and there are $C, c, B>0$ satisfying

$$
\left|\partial_{\xi}^{\alpha} \partial_{x}^{\beta} p_{j}(x, \xi)\right| \leq C^{|\alpha|+|\beta|+2 j+1} \alpha !^{\mu} \beta !^{v} j !^{\mu+v-1}\langle\xi\rangle^{m_{1}-\rho_{1}|\alpha|+\delta_{1}|\beta|-k_{j}}\langle x\rangle^{m_{2}-\rho_{2}|\beta|+\delta_{2}|\alpha|-\ell_{j}}
$$

for $\alpha, \beta \in \mathbb{N}_{0}^{n}, j \geq 0$ and $(x, \xi) \in Q_{B_{2}(j), B_{1}(j)^{\prime}}^{e}$ where $B_{i}(j)=\left(B j^{\mu+v-1}\right)^{\frac{1}{\Lambda_{i}}}, i=1,2$. 
Definition 5. Given $\sum_{j \geq 0} p_{j}, \sum_{j \geq 0} q_{j} \in F_{\bar{\sigma}} S G_{\mu, v}^{m}$, we say that $\sum_{j \geq 0} p_{j} \sim \sum_{j \geq 0} q_{j}$ in $F_{\bar{\sigma}} S G_{\rho, \delta ;}^{m}(\mu, v)$ if there are $C, c, B>0$ satisfying

$$
\left|\partial_{\xi}^{\alpha} \partial_{x}^{\beta} \sum_{j<N}\left(p_{j}-q_{j}\right)(x, \xi)\right| \leq C^{|\alpha|+|\beta|+2 N+1} \alpha !^{\mu} \beta !^{\nu} N !^{\mu+v-1}\langle\xi\rangle^{m_{1}-\rho_{1}|\alpha|+\delta_{1}|\beta|-k_{N}}\langle x\rangle^{m_{2}-\rho_{2}|\beta|+\delta_{2}|\alpha|-\ell_{N}}
$$

for $\alpha, \beta \in \mathbb{N}_{0}^{n}, N \geq 1$ and $(x, \xi) \in Q_{B_{2}(N), B_{1}(N)}^{e}$.

Remark 1. If $k_{j}=\left(\rho_{1}-\delta_{1}\right) j, \ell_{j}=\left(\rho_{2}-\delta_{2}\right) j$ and $\Lambda_{i}=\rho_{i}-\delta_{i}, i=1,2$, we simply write $\operatorname{FSG}_{\rho, \delta ;(\mu, v)}^{m}$ and we recover the usual definitions presented under different notation in [23]. If moreover $\rho=(1,1), \delta=(0,0)$, we use the notation $\operatorname{FSG}_{(\mu, v)}^{m}$.

Remark 2. If $\sum_{j \geq 0} p_{j} \in F_{\bar{\sigma}} S G_{\rho, \delta ;(\mu, v)}^{m}$, then $p_{0} \in S G_{\rho, \delta ;(\mu, v)}^{m}$. Given $p \in S G_{\rho, \delta ;(\mu, v)}^{m}$ and setting $p_{0}=p, p_{j}=0$, $j \geq 1$, we have $p=\sum_{j \geq 0} p_{j}$. Hence, we can consider $S G_{\rho, \delta ;(\mu, v)}^{m}$ as a subset of $F_{\bar{\sigma}} S G_{\rho, \delta ;(\mu, v)}^{m}$.

Proposition 1. Given $\sum_{j \geq 0} p_{j} \in F_{\bar{\sigma}} S G_{\rho, \delta ;(\mu, v)}^{m}$, there exists $p \in S G_{\rho, \delta ;(\mu, v)}^{m}$ such that $p \sim \sum_{j \geq 0} p_{j}$ in $F_{\bar{\sigma}} S G_{\rho, \delta ;(\mu, v)}^{m}$.

Proposition 2. Let $p \in S G_{\rho, \delta ;(\mu, v)}^{(0,0)}$ such that $p \sim 0$ in $F_{\bar{\sigma}} S G_{\rho, \delta ;(\mu, v)}^{(0,0)}$. Then, $p \in \mathcal{S}_{r}\left(\mathbb{R}^{2 n}\right)$ for $r \geq \max \left\{\frac{1}{\tilde{\Lambda}}(\mu+\right.$ $v-1), \mu+v-1\}$, where $\tilde{\Lambda}=\min \left\{\Lambda_{1}, \Lambda_{2}\right\}$.

Proposition 3. Let $p \in S G_{\rho, \delta ;(\mu, v)}^{m}$ and let $P^{*}$ be the $L^{2}$ adjoint of $p(x, D)$. Then, there exists a symbol $p^{*} \in S G_{\rho, \delta ;(u, v)}^{m}$ such that $P^{*}=p^{*}(x, D)+R$, where $R$ is a $\mathcal{S}_{r}$-regularizing operator for any $r \geq \frac{\mu+v-1}{\min \left\{\varrho_{1}-\delta_{1}, \varrho_{2}-\delta_{2}\right\}}$. Moreover,

$$
p^{*}(x, \xi) \sim \sum_{j \geq 0} \sum_{|\alpha|=j} \alpha !^{-1} \partial_{\xi}^{\alpha} D_{x}^{\alpha} \overline{p(x, \xi)} \quad \text { in } \quad F S G_{\rho, \delta ;(\mu, v)}^{m}
$$

Proposition 4. Let $p \in S G_{\rho, \delta ;(\mu, v)}^{m}, q \in S G_{\rho, \delta ;(\mu, v)}^{m^{\prime}}$. Then, there exists a symbol $s \in S G_{\rho, \delta ;(\mu, v)}^{m+m^{\prime}}$ such that $p(x, D) q(x, D)=s(x, D)+R^{\prime}$ where $R^{\prime}$ is a $\mathcal{S}_{r}$-regularizing operator for any $r \geq \frac{\mu+v-1}{\min \left\{\varrho_{1}-\delta_{1}, \varrho_{2}-\delta_{2}\right\}}$. Moreover,

$$
s(x, \xi) \sim \sum_{j \geq 0} \sum_{|\alpha|=j} \alpha !^{-1} \partial_{\xi}^{\alpha} p(x, \xi) D_{x}^{\alpha} q(x, \xi) \quad \text { in } \quad F S G_{\rho, \delta ;(\mu, v)}^{m+m^{\prime}} .
$$

\section{Oscillatory Integrals and Operators with Double Symbols}

To define the Friedrichs part of an operator, it is necessary to extend the notion of pseudodifferential operator as in [5] by considering more general symbols called double symbols. Quantizations of these symbols are defined as oscillatory integrals.

\subsection{Amplitudes and Oscillatory Integrals}

Definition 6 (Amplitudes). For $m \in \mathbb{R}^{2}$ and $\delta \in[0,1)^{2}$, we define $\mathcal{A}_{\delta}^{m}\left(\mathbb{R}^{2 n}\right)$ as the space of all smooth functions $a(\eta, y)$ such that

$$
\left|\partial_{\eta}^{\alpha} \partial_{y}^{\beta} a(\eta, y)\right| \leq C_{\alpha, \beta}\langle\eta\rangle^{m_{1}+\delta_{1}|\beta|}\langle y\rangle^{m_{2}+\delta_{2}|\alpha|}, \quad \eta, y \in \mathbb{R}^{n} .
$$

For $\ell \in \mathbb{N}_{0}$ and $a \in \mathcal{A}_{\delta}^{m}\left(\mathbb{R}^{2 n}\right)$, the seminorms

$$
|a|_{\ell}=\max _{|\alpha+\beta| \leq \ell} \sup _{\eta, y \in \mathbb{R}^{n}}\left\{\left|\partial_{\eta}^{\alpha} \partial_{y}^{\beta} a(\eta, y)\right|\langle\eta\rangle^{-\left(m_{1}+\delta_{1}|\beta|\right)}\langle y\rangle^{-\left(m_{2}+\delta_{2}|\alpha|\right)}\right\},
$$


$\operatorname{turn} \mathcal{A}_{\delta}^{m}\left(\mathbb{R}^{2 n}\right)$ into a Fréchet space.

Remark 3. In [5] (Chapter 1, Section 6), the special case $\mathcal{A}_{(\delta, 0)}^{(m, \tau)}\left(\mathbb{R}^{n}\right)$, where $m \in \mathbb{R}, \tau>0$ and $\delta \in[0,1)$, is treated.

Definition 7 (Oscillatory Integral). For $a \in \mathcal{A}_{\delta, \tau}^{m}$, we define

$$
\begin{aligned}
O s-\left[e^{-i \eta y} a(\eta, y)\right] & =O s-\iint e^{-i \eta y} a(\eta, y) d y d \eta \\
& :=\lim _{\varepsilon \rightarrow 0} \iint e^{-i \eta y} \chi_{\varepsilon}(\eta, y) a(\eta, y) d y d \eta
\end{aligned}
$$

where $\chi_{\varepsilon}(\eta, y)=\chi(\varepsilon \eta, \varepsilon y)$ and $\chi$ is a Schwartz function on $\mathbb{R}^{2 n}$ such that $\chi(0,0)=1$.

Theorem 1. Let $a \in \mathcal{A}_{\delta}^{m}\left(\mathbb{R}^{2 n}\right)$. If $\ell, \ell^{\prime} \in \mathbb{N}_{0}$ satisfy

$$
-2 \ell\left(1-\delta_{1}\right)+m_{1}<-n, \quad-2 \ell^{\prime}\left(1-\delta_{2}\right)+m_{2}<-n,
$$

then $\left|\langle y\rangle^{-2 \ell^{\prime}}\left\langle D_{\eta}\right\rangle^{2 \ell^{\prime}}\left\{\langle\eta\rangle^{-2 \ell}\left\langle D_{y}\right\rangle^{2 \ell} a(\eta, y)\right\}\right|$ belongs to $L^{1}\left(\mathbb{R}^{2 n}\right)$ and we have

$$
\text { Os }-\left[e^{-i \eta y} a(\eta, y)\right]=\iint e^{-i \eta y}\langle y\rangle^{-2 \ell^{\prime}}\left\langle D_{\eta}\right\rangle^{2 \ell^{\prime}}\left\{\langle\eta\rangle^{-2 \ell}\left\langle D_{y}\right\rangle^{2 \ell} a(\eta, y)\right\} d y d \eta \text {. }
$$

Furthermore, there is $C_{\ell, \ell^{\prime}}>0$ independent of $a \in \mathcal{A}_{\delta, \tau}^{m}\left(\mathbb{R}^{2 n}\right)$ such that

$$
\left|O s-\left[e^{-i \eta y} a(\eta, y)\right]\right| \leq C_{\ell, \ell^{\prime}}|a|_{2\left(\ell+\ell^{\prime}\right)} .
$$

Proof. Integration by parts gives

$$
\text { Os }-\left[e^{-i \eta y} a\right]=\lim _{\varepsilon \rightarrow 0} \iint e^{-i \eta y}\langle y\rangle^{-2 \ell^{\prime}}\left\langle D_{\eta}\right\rangle^{2 \ell^{\prime}}\left\{\langle\eta\rangle^{-2 \ell}\left\langle D_{y}\right\rangle^{2 \ell}\left(a \chi_{\varepsilon}\right)\right\} d y d \eta,
$$

where $\chi_{\varepsilon}(\eta, y)=\chi(\varepsilon \eta, \varepsilon y)$. Since

$$
\left\langle D_{\eta}\right\rangle^{2 \ell^{\prime}}=\sum_{\ell_{0}^{\prime}+\left|L^{\prime}\right|=\ell^{\prime}} \frac{\ell^{\prime} !}{\ell_{0}^{\prime} ! L^{\prime} !} D_{\eta}^{2 L^{\prime}}, \quad\left\langle D_{y}\right\rangle^{2 \ell}=\sum_{\ell_{0}+|L|=\ell} \frac{\ell !}{\ell_{0} ! L !} D_{y}^{2 L},
$$

where $L^{\prime}=\left(\ell_{1}^{\prime}, \ldots, \ell_{n}^{\prime}\right)$ and $L=\left(\ell_{1}, \ldots, \ell_{n}\right)$, we have

$$
\begin{aligned}
& \left\langle D_{\eta}\right\rangle^{2 \ell^{\prime}}\left\{\langle\eta\rangle^{-2 \ell}\left\langle D_{y}\right\rangle^{2 \ell}\left(\chi_{\varepsilon} a\right)\right\}=\sum_{\ell_{0}^{\prime}+\left|L^{\prime}\right|=\ell^{\prime}} \frac{\ell^{\prime} !}{\ell_{0}^{\prime} ! L^{\prime} !} D_{\eta}^{2 L^{\prime}}\left\{\langle\eta\rangle^{-2 \ell}\left\langle D_{y}\right\rangle^{2 \ell}\left(\chi_{\varepsilon} a\right)\right\} \\
& =\sum_{\ell_{0}^{\prime}+\left|L^{\prime}\right|=\ell^{\prime}} \frac{\ell^{\prime} !}{\ell_{0}^{\prime} ! L^{\prime} !} \sum_{\alpha_{1}+\alpha_{2}=2 L^{\prime}} \frac{\left(2 L^{\prime}\right) !}{\alpha_{1} ! \alpha_{2} !} D_{\eta}^{\alpha_{1}}\langle\eta\rangle^{-2 \ell} \cdot D_{\eta}^{\alpha_{2}}\left\langle D_{y}\right\rangle^{2 \ell}\left(\chi_{\varepsilon} a\right) \\
& =\sum_{\ell_{0}^{\prime}+\left|L^{\prime}\right|=\ell^{\prime}} \frac{\ell^{\prime} !}{\ell_{0}^{\prime} ! L^{\prime} !} \sum_{\alpha_{1}+\alpha_{2}=2 L^{\prime}} \frac{\left(2 L^{\prime}\right) !}{\alpha_{1} ! \alpha_{2} !} D_{\eta}^{\alpha_{1}}\langle\eta\rangle^{-2 \ell} \\
& \times \sum_{\ell_{0}+|L|=\ell} \frac{\ell !}{\ell_{0} ! L !} D_{\eta}^{\alpha_{2}} D_{y}^{2 L}\left(\chi_{\varepsilon} a\right) \\
& =\sum_{\ell_{0}^{\prime}+\left|L^{\prime}\right|=\ell^{\prime}} \frac{\ell^{\prime} !}{\ell_{0}^{\prime} ! L^{\prime} !} \sum_{\alpha_{1}+\alpha_{2}=2 L^{\prime}} \frac{\left(2 L^{\prime}\right) !}{\alpha_{1} ! \alpha_{2} !} D_{\eta}^{\alpha_{1}}\langle\eta\rangle^{-2 \ell} \sum_{\ell_{0}+|L|=\ell} \frac{\ell !}{\ell_{0} ! L !} \\
& \times \sum_{\alpha_{1}^{\prime}+\alpha_{2}^{\prime}=\alpha_{2}} \sum_{\beta_{1}+\beta_{2}=2 L} \frac{\alpha_{2} !}{\alpha_{1}^{\prime} ! \alpha_{2}^{\prime} !} \frac{(2 L) !}{\beta_{1} ! \beta_{2} !} D_{\eta}^{\alpha_{1}^{\prime}} D_{y}^{\beta_{1}} \chi_{\varepsilon} D_{\eta}^{\alpha_{2}^{\prime}} D_{y}^{\beta_{2}} a \text {. }
\end{aligned}
$$


Hence, we obtain the following estimates, for $\varepsilon$ in $[0,1]$,

$$
\begin{aligned}
& \left|\left\langle D_{\eta}\right\rangle^{2 \ell^{\prime}}\left\{\langle\eta\rangle^{-2 \ell}\left\langle D_{y}\right\rangle^{2 \ell}\left(\chi_{\varepsilon} a\right)\right\}\right| \leq \sum_{\ell_{0}^{\prime}+\left|L^{\prime}\right|=\ell^{\prime}} \frac{\ell^{\prime} !}{\ell_{0}^{\prime} ! L^{\prime} !} \sum_{\alpha_{1}+\alpha_{2}=2 L^{\prime}} \frac{\left(2 L^{\prime}\right) !}{\alpha_{1} ! \alpha_{2} !} \\
& \times C_{0}^{\left|\alpha_{1}\right|+1} \alpha_{1} !\langle\eta\rangle^{-2 \ell-\left|\alpha_{1}\right|} \sum_{\ell_{0}+|L|=\ell} \frac{\ell !}{\ell_{0} ! L !} \sum_{\alpha_{1}^{\prime}+\alpha_{2}^{\prime}=\alpha_{2} \beta_{1}+\beta_{2}=2 L} \sum_{\frac{\alpha_{2} !}{\alpha_{1}^{\prime} ! \alpha_{2}^{\prime} !} \frac{(2 L) !}{\beta_{1} ! \beta_{2} !}} \times \varepsilon^{\left|\alpha_{1}^{\prime}+\beta_{1}\right|} C_{\chi}^{\left|\alpha_{1}^{\prime}\right|+\left|\beta_{1}\right|+1} \alpha_{1}^{\prime} !^{\mu} \beta_{1} !^{v}|a|_{\left|\alpha_{2}^{\prime}+\beta_{2}\right|}\langle\eta\rangle^{m_{1}+\delta_{1}\left|\beta_{2}\right|}\langle y\rangle^{m_{2}+\delta_{2}\left|\alpha_{2}^{\prime}\right|} \\
& \leq C_{\ell, \ell^{\prime}}|a|_{2\left(\ell+\ell^{\prime}\right)}\langle\eta\rangle^{m-2 \ell\left(1-\delta_{1}\right)}\langle y\rangle^{m_{2}+2 \ell^{\prime} \delta_{2}}
\end{aligned}
$$

and

$$
\begin{aligned}
\langle y\rangle^{-2 \ell^{\prime}} \mid\left\langle D_{\eta}\right\rangle^{2 \ell^{\prime}}\left\{\langle\eta\rangle^{-2 \ell}\right. & \left.\left\langle D_{y}\right\rangle^{2 \ell}\left(\chi_{\varepsilon} a\right)\right\} \mid \\
& \leq C_{\ell, \ell^{\prime}}|a|_{2\left(\ell+\ell^{\prime}\right)}\langle\eta\rangle^{m_{1}-2 \ell\left(1-\delta_{1}\right)}\langle y\rangle^{m_{2}-2 \ell^{\prime}\left(1-\delta_{2}\right)} .
\end{aligned}
$$

Finally, by Lemma 6.3 on Page 47 of [5] and Lebesgue dominated convergence theorem, we obtain

$$
\begin{gathered}
O s-\left[e^{-i \eta y} a\right]=\iint e^{-i \eta y}\langle y\rangle^{-2 \ell^{\prime}}\left\langle D_{\eta}\right\rangle^{2 \ell^{\prime}}\left\{\langle\eta\rangle^{-2 \ell}\left\langle D_{y}\right\rangle^{2 \ell} a\right\} d y d \eta, \\
\left|O s-\left[e^{-i \eta y} a\right]\right| \leq C_{\ell, \ell^{\prime}}|a|_{2\left(\ell+\ell^{\prime}\right)} \iint\langle\eta\rangle^{m_{1}-2 \ell\left(1-\delta_{1}\right)}\langle y\rangle^{m_{2}-2 \ell^{\prime}\left(1-\delta_{2}\right)} d y d \eta .
\end{gathered}
$$

Following the ideas in the proofs of Theorems 6.7 and 6.8 of [5] (Chapter 1, Section 6), one can obtain the following result.

Proposition 5. Let $a \in \mathcal{A}_{\delta}^{m}\left(\mathbb{R}^{2 n}\right), \alpha, \beta \in \mathbb{N}_{0}$ and $\eta_{0}, y_{0} \in \mathbb{R}^{n}$. Then,

(i) Os $-\left[e^{-i \eta y} y^{\alpha} a\right]=O s-\left[\left(-D_{\eta}\right)^{\alpha} e^{-i \eta y} a\right]=O s-\left[e^{-i \eta y} D_{\eta}^{\alpha} a\right]$;

(ii) $O s-\left[e^{-i \eta y} \eta^{\beta} a\right]=O s-\left[\left(-D_{y}\right)^{\beta} e^{-i \eta y} a\right]=O s-\left[e^{-i \eta y} D_{y}^{\beta} a\right]$; and

(iii) Os $-\left[e^{-i \eta y} a(\eta, y)\right]=O s-\left[e^{-i\left(\eta-\eta_{0}\right)\left(y-y_{0}\right)} a\left(\eta-\eta_{0}, y-y_{0}\right)\right]$.

3.2. Operators with Double Symbols

Definition 8. Let $m=\left(m_{1}, m_{2}\right), m^{\prime}=\left(m_{1}^{\prime}, m_{2}^{\prime}\right) \in \mathbb{R}^{2}$ and $\rho=\left(\rho_{1}, \rho_{2}\right), \delta=\left(\delta_{1}, \delta_{2}\right)$ such that $0 \leq \delta_{j}<$ $\rho_{j} \leq 1, j=1,2$. We denote by $S G_{\rho, \delta}^{m, m^{\prime}}\left(\mathbb{R}^{4 n}\right)$ the space of all functions $p \in C^{\infty}\left(\mathbb{R}^{4 n}\right)$ such that for any $\alpha, \alpha^{\prime}, \beta, \beta^{\prime} \in \mathbb{N}_{0}^{n}$ there is $C_{\alpha, \beta}^{\alpha^{\prime}, \beta^{\prime}}>0$ for which

$$
\left|p_{\beta, \beta^{\prime}}^{\alpha, \alpha^{\prime}}\left(x, \xi, x^{\prime}, \xi^{\prime}\right)\right| \leq C_{\alpha, \beta}^{\alpha^{\prime}, \beta^{\prime}}\langle\xi\rangle^{m_{1}-\rho_{1}|\alpha|}\left\langle\xi^{\prime}\right\rangle^{m_{1}^{\prime}-\rho_{1}\left|\alpha^{\prime}\right|}\left\langle\xi ; \xi^{\prime}\right\rangle^{\delta_{1}\left|\beta+\beta^{\prime}\right|}\langle x\rangle^{m_{2}-\rho_{2}|\beta|}\left\langle x^{\prime}\right\rangle^{m_{2}^{\prime}-\rho_{2}\left|\beta^{\prime}\right|}\left\langle x ; x^{\prime}\right\rangle^{\delta_{2}\left|\alpha+\alpha^{\prime}\right|}
$$

for every $x, x^{\prime}, \xi, \xi^{\prime} \in \mathbb{R}^{n}$, where $p_{\beta, \beta^{\prime}}^{\alpha, \alpha^{\prime}}=\partial_{\tilde{\xi}}^{\alpha} \partial_{\xi^{\prime}}^{\alpha^{\prime}} D_{x}^{\beta} D_{x^{\prime}}^{\beta^{\prime}} p$ and $\left\langle z ; z^{\prime}\right\rangle=\sqrt{1+|z|^{2}+\left|z^{\prime}\right|^{2}}$ for $z, z^{\prime} \in \mathbb{R}^{n}$.

Denoting by $|p|_{\alpha, \alpha^{\prime}, \beta, \beta^{\prime}}^{m, m^{\prime}}$ the supremum over $x, \xi, x^{\prime}, \xi^{\prime} \in \mathbb{R}^{n}$ of

$$
\left|p_{\beta, \beta^{\prime}}^{\alpha, \alpha^{\prime}}\left(x, \xi, x^{\prime}, \xi^{\prime}\right)\right|\langle\xi\rangle^{-m_{1}+\rho_{1}|\alpha|}\left\langle\xi^{\prime}\right\rangle^{-m_{1}^{\prime}+\rho_{1}\left|\alpha^{\prime}\right|}\left\langle\xi^{\prime} ; \xi^{\prime}\right\rangle^{-\delta_{1}\left|\beta+\beta^{\prime}\right|}\langle x\rangle^{-m_{2}+\rho_{2}|\beta|}\left\langle x^{\prime}\right\rangle^{-m_{2}^{\prime}+\rho_{2}\left|\beta^{\prime}\right|}\left\langle x ; x^{\prime}\right\rangle^{-\delta_{2}\left|\alpha+\alpha^{\prime}\right|}
$$

the space $\mathbf{S G}_{\rho, \delta}^{m, m^{\prime}}$ is a Fréchet space whose topology is defined by the family of seminorms

$$
|p|_{\ell}^{m, m^{\prime}}:=\sup _{\left|\alpha+\beta+\alpha^{\prime}+\beta^{\prime}\right| \leq \ell}|p|_{\alpha, \alpha^{\prime}, \beta, \beta^{\prime}}^{m, m^{\prime}}
$$


Definition 9. Let $m=\left(m_{1}, m_{2}\right), m^{\prime}=\left(m_{1}^{\prime}, m_{2}^{\prime}\right) \in \mathbb{R}^{2}, \rho=\left(\rho_{1}, \rho_{2}\right), \delta=\left(\delta_{1}, \delta_{2}\right)$ such that $0 \leq \delta_{j}<\rho_{j} \leq 1, j=1,2$, and let $\mu, v \geq 1$. We denote by $S G_{\rho, \delta ;(\mu, v)}^{m, m^{\prime}}\left(\mathbb{R}^{4 n}\right)$ the space of all functions $p \in C^{\infty}\left(\mathbb{R}^{4 n}\right)$ such that for some $C>0$ :

$$
\begin{aligned}
\left|p_{\beta, \beta^{\prime}}^{\alpha, \alpha^{\prime}}\left(x, \xi, x^{\prime}, \xi^{\prime}\right)\right| & \leq C^{\left|\alpha+\beta+\alpha^{\prime}+\beta^{\prime}\right|}\left(\alpha ! \alpha^{\prime} !\right)^{\mu}\left(\beta ! \beta^{\prime} !\right)^{v}\langle\xi\rangle^{m_{1}-\rho_{1}|\alpha|}\left\langle\xi^{\prime}\right\rangle^{m_{1}^{\prime}-\rho_{1}\left|\alpha^{\prime}\right|}\left\langle\xi ; \xi^{\prime}\right\rangle^{\delta_{1}\left|\beta+\beta^{\prime}\right|} \\
& \times\langle x\rangle^{m_{2}-\rho_{2}|\beta|}\left\langle x^{\prime}\right\rangle^{m_{2}^{\prime}-\rho_{2}\left|\beta^{\prime}\right|}\left\langle x ; x^{\prime}\right\rangle^{\delta_{2}\left|\alpha+\alpha^{\prime}\right|} .
\end{aligned}
$$

For $C>0$, the space $\mathbf{S G}_{\rho, \delta ;(\mu, v)}^{m, m^{\prime}}\left(\mathbb{R}^{4 n} ; C\right)$ of all smooth functions $p\left(x, \xi, x^{\prime}, \xi^{\prime}\right)$, such that (9) holds for a fixed $C>0$, is a Banach space with norm

$$
|p|_{C}^{m, m^{\prime}}:=\sup _{\alpha, \alpha^{\prime}, \beta, \beta^{\prime} \in \mathbb{N}_{0}^{n}} C^{-\left|\alpha+\alpha^{\prime}+\beta+\beta^{\prime}\right|}\left(\alpha ! \alpha^{\prime} !\right)^{-\mu}\left(\beta ! \beta^{\prime} !\right)^{-v}|p|_{\alpha, \alpha^{\prime}, \beta, \beta^{\prime}}^{m, m^{\prime}}
$$

After that, we define $\mathbf{S G}_{\rho, \delta ;(\mu, v)}^{m, m^{\prime}}=\bigcup_{C>0} \mathbf{S G}_{\rho, \delta ;(\mu, v)}^{m, m^{\prime}}\left(\mathbb{R}^{4 n} ; C\right)$ as an inductive limit of Banach spaces.

Definition 10. For $p \in S G_{\rho, \delta}^{m, m^{\prime}}$, we define

$$
\begin{aligned}
p\left(x, D_{x}, x^{\prime}, D_{x^{\prime}}\right) u(x) & :=\int e^{i \xi\left(x-x^{\prime}\right)} e^{i \xi^{\prime}\left(x^{\prime}-x^{\prime \prime}\right)} p\left(x, \xi, x^{\prime}, \xi^{\prime}\right) u\left(x^{\prime \prime}\right) d x^{\prime \prime} d \xi^{\prime} d x^{\prime} d \xi \\
& =\int e^{i \xi\left(x-x^{\prime}\right)} e^{i \xi^{\prime} x^{\prime}} p\left(x, \xi, x^{\prime}, \xi^{\prime}\right) \widehat{u}\left(\xi^{\prime}\right) d \xi^{\prime} d x^{\prime} d \xi
\end{aligned}
$$

for every $u \in \mathscr{S}\left(\mathbb{R}^{n}\right)$.

Lemma 1. Let $p \in S G_{\rho,\left(0, \delta_{2}\right)}^{m, m^{\prime}}\left(\mathbb{R}^{4 n}\right)$. For any multi-indices $\alpha, \alpha^{\prime}, \beta, \beta^{\prime}$, set $q=p_{\beta, \beta^{\prime}}^{\alpha, \alpha^{\prime}}$ and, for $\theta \in[-1,1]$, define

$$
q_{\theta}(x, \xi)=O s-\iint e^{-i \eta y} q(x, \xi+\theta \eta, x+y, \xi) d y d \eta, \quad x, \xi \in \mathbb{R}^{n} .
$$

Then, $\left\{q_{\theta}\right\}_{|\theta| \leq 1}$ is bounded in $S G_{\rho,\left(0, \delta_{2}\right)}^{\tau}\left(\mathbb{R}^{2 n}\right)$, where $\tau=\left(\tau_{1}, \tau_{2}\right), \tau_{1}=m_{1}+m_{1}^{\prime}-\rho_{1}\left|\alpha+\alpha^{\prime}\right|, \tau_{2}=$ $m_{2}+m_{2}^{\prime}-\rho_{2}\left|\beta+\beta^{\prime}\right|+\delta_{2}\left|\alpha+\alpha^{\prime}\right|$. Furthermore, for any $\ell \in \mathbb{N}_{0}$, there are $\ell^{\prime}:=\ell^{\prime}(\ell) \in \mathbb{N}_{0}$ and $C_{\ell, \ell^{\prime}}>0$ independent of $\theta$ such that

$$
\left|q_{\theta}\right|_{\ell}^{\tau} \leq C_{\ell, \ell^{\prime}}|p|_{\ell^{\prime}}^{m, m^{\prime}}
$$

Proof. First, notice that $q(x, \xi+\theta \eta, x+y, \xi) \in \mathcal{A}_{\left(0, \delta_{2}\right)}^{\left(m_{1}, m_{2}^{\prime}+\delta_{2}\left|\alpha+\alpha^{\prime}\right|\right)}\left(\mathbb{R}_{\eta, y}^{2 n}\right)$, therefore $q_{\theta}(x, \xi)$ is well defined for any fixed $\xi, x, \theta$. Given $\gamma, \mu \in \mathbb{N}_{0}^{n}$, we may write, omitting the variables $(x, \xi+\theta \eta, x+y, \xi)$,

$$
\partial_{x}^{\gamma} \partial_{\tilde{\zeta}}^{\mu} q=\sum_{\substack{\mu^{\prime} \leq \mu \\ \gamma^{\prime} \leq \gamma}} \frac{\mu !}{\mu^{\prime} !\left(\mu-\mu^{\prime}\right) !} \frac{\gamma !}{\gamma^{\prime} !\left(\gamma-\gamma^{\prime}\right) !} p_{\left(\beta+\gamma^{\prime}, \beta^{\prime}+\gamma-\gamma^{\prime}\right)}^{\left(\alpha+\mu^{\prime}, \alpha^{\prime}+\mu-\mu^{\prime}\right)} .
$$

To prove that $\left\{q_{\theta}\right\}_{|\theta| \leq 1}$ is bounded in $\mathbf{S G}_{\rho,\left(0, \delta_{2}\right)}^{\tau}\left(\mathbb{R}^{2 n}\right)$, it is sufficient to show that

$$
\left|q_{\theta}(x, \xi)\right| \leq C_{1}|p|_{\ell_{1}}^{m, m^{\prime}}\langle\xi\rangle^{\tau_{1}}\langle x\rangle^{\tau_{2}}
$$


for some $C_{1}>0$ and $\ell_{1} \in \mathbb{N}_{0}$ depending on $\alpha, \beta, \alpha^{\prime}, \beta^{\prime}$. Indeed, if (11) holds, then we can estimate the derivatives of $q_{\theta}$ as follows:

$$
\begin{aligned}
& \left|\partial_{x}^{\gamma} \partial_{\xi}^{\mu} q_{\theta}(x, \xi)\right|=\left|O s-\iint e^{-i y \eta} \partial_{x}^{\gamma} \partial_{\xi}^{\mu} q(x, \xi+\theta \eta, x+y, \xi) d y d \eta\right| \\
& \leq \sum_{\substack{\gamma^{\prime} \leq \gamma \\
\mu^{\prime} \leq \mu}}\left(\begin{array}{c}
\mu \\
\mu^{\prime}
\end{array}\right)\left(\begin{array}{c}
\gamma \\
\gamma^{\prime}
\end{array}\right)\left|O s-\iint e^{-i y \eta} p_{\left(\beta+\gamma^{\prime}, \beta^{\prime}+\gamma-\gamma^{\prime}\right)}^{\left(\alpha+\mu^{\prime}, \alpha^{\prime}+\mu-\mu^{\prime}\right)}(x, \xi+\theta \eta, x+y, \xi) d y d \eta\right| \\
& \leq \sum_{\substack{\gamma^{\prime} \leq \gamma \\
\mu^{\prime} \leq \mu}}\left(\begin{array}{c}
\mu \\
\mu^{\prime}
\end{array}\right)\left(\begin{array}{c}
\gamma \\
\gamma^{\prime}
\end{array}\right) C_{1}\left(\alpha, \alpha^{\prime}, \beta, \beta^{\prime}, \gamma, \mu\right)|p|_{\ell_{1}}^{m, m^{\prime}} \\
& \times\langle\bar{\zeta}\rangle^{m_{1}+m_{1}^{\prime}-\rho_{1}\left|\alpha+\mu^{\prime}+\alpha^{\prime}+\mu-\mu^{\prime}\right|}\langle x\rangle^{m_{2}+m_{2}^{\prime}-\rho_{2}\left|\beta+\gamma^{\prime}+\beta^{\prime}+\gamma-\gamma^{\prime}\right|+\delta_{2}\left|\alpha+\mu^{\prime}+\alpha^{\prime}+\mu-\mu^{\prime}\right|} \\
& \leq C|p|_{\ell_{1}}^{m, m^{\prime}}\langle\bar{\zeta}\rangle^{m_{1}+m_{1}^{\prime}-\rho_{1}\left|\alpha+\alpha^{\prime}+\mu\right|}\langle x\rangle^{m_{2}+m_{2}^{\prime}-\rho_{2}\left|\beta+\beta^{\prime}+\gamma\right|+\delta_{2}\left|\alpha+\alpha^{\prime}+\mu\right|}
\end{aligned}
$$

where $C$ and $\ell_{1}$ depend of $\alpha, \alpha^{\prime}, \beta, \beta^{\prime}, \gamma, \mu$ and does not depend of $\theta$.

Now, we show that (11) holds true. Observe that

$$
e^{-i \eta y}=\left(1+\langle x\rangle^{2 \delta_{2}}|\eta|^{2}\right)^{-\ell}\left(1-\langle x\rangle^{2 \delta_{2}} \Delta_{y}\right)^{\ell} e^{-i \eta y},
$$

therefore

$$
q_{\theta}(x, \xi)=O s-\iint e^{-i y \eta} r_{\theta}(x, \xi ; \eta, y) d y d \eta
$$

where

$$
r_{\theta}(x, \xi ; \eta, y)=\left(1+\langle x\rangle^{2 \delta_{2}}|\eta|^{2}\right)^{-\ell}\left(1-\langle x\rangle^{2 \delta_{2}} \Delta_{y}\right)^{\ell} q(x, \xi+\theta \eta, x+y, \xi) .
$$

If we take $\ell$ satisfying $2 \ell>\left|m_{1}\right|+n$, then $r_{\theta}$ is integrable with respect to $\eta$. Now, consider a cutoff function $\chi(y)$ such that $\chi(y)=1$ for $|y| \leq 4^{-1}\langle x\rangle$ and $\chi(y)=0$ for $|y| \geq 2^{-1}\langle x\rangle$. Then, we can write

$$
\text { Os }-\iint e^{-i y \eta} r_{\theta}(x, \xi ; \eta, y) d y d \eta=I_{1}+I_{2}+I_{3},
$$

where

$$
\begin{gathered}
I_{1}=\int_{\mathbb{R}_{\eta}^{n}} \int_{|y| \leq 4^{-1}\langle x\rangle^{\delta_{2}}} e^{-i y \eta} r_{\theta}(x, \xi ; \eta, y) \chi(y) d y d \eta, \\
I_{2}=\int_{\mathbb{R}_{\eta}^{n}} \int_{4^{-1}\langle x\rangle^{\delta_{2}} \leq|y| \leq 2^{-1}\langle x\rangle} e^{-i y \eta} r_{\theta}(x, \xi ; \eta, y) \chi(y) d y d \eta, \\
I_{3}=O s-\left[e^{-i y \eta} r_{\theta}(x, \xi ; \eta, y)(1-\chi(y))\right] .
\end{gathered}
$$

Let us obtain a useful inequality when $|y| \leq 2^{-1}\langle x\rangle$. Since

$$
|\langle x+y\rangle-\langle x\rangle| \leq \int_{0}^{1}\left|\frac{d}{d t}\langle x+t y\rangle\right| d t \leq \int_{0}^{1}|y| \frac{|x+t y|}{\langle x+t y\rangle} d t \leq|y|,
$$

for $|y| \leq 2^{-1}\langle x\rangle$, we have

$$
\frac{1}{2}\langle x\rangle \leq\langle x+y\rangle \leq \frac{3}{2}\langle x\rangle, \quad\langle x ; x+y\rangle \leq\langle x\rangle+|x+y| \leq \frac{5}{2}\langle x\rangle .
$$


Now, we proceed to estimate $I_{1}, I_{2}, I_{3}$. We begin with $I_{1}$. With aid of Petree's inequality and using the fact that $\rho_{2}>\delta_{2}$, we obtain, for $|y| \leq 4^{-1}\langle x\rangle^{\delta_{2}}$ and $|\theta| \leq 1$,

$$
\begin{aligned}
& \left|r_{\theta}(x, \xi ; \eta, y)\right| \leq\left(1+\langle x\rangle^{2 \delta_{2}}|\eta|^{2}\right)^{-\ell} \sum_{\ell_{0}+|L|=\ell} \frac{\ell !}{\ell_{0} ! L !}\langle x\rangle^{2 \delta_{2}|L|}\left|p_{\left(\beta, \beta^{\prime}+2 L\right)}^{\left(\alpha, \alpha^{\prime}\right)}\right| \\
& \quad \leq\left(1+\langle x\rangle^{2 \delta_{2}}|\eta|^{2}\right)^{-\ell}\langle x\rangle^{2 \delta_{2}|L|} \sum_{\ell_{0}+|L|=\ell} \frac{\ell !}{\ell_{0} ! L !}|p|_{\left|\alpha+\alpha^{\prime}+\beta+\beta^{\prime}\right|+2 \ell}^{m, m^{\prime}} \\
& \quad \times\langle\xi+\theta \eta\rangle^{m_{1}-\rho_{1}|\alpha|}\langle\xi\rangle^{m_{1}^{\prime}-\rho_{1}\left|\alpha^{\prime}\right|}\langle x\rangle^{m_{2}-\rho_{2}|\beta|}\langle x+y\rangle^{m_{2}^{\prime}-\rho_{2}\left|\beta^{\prime}+2 L\right|}\langle x ; x+y\rangle^{\delta_{2}\left|\alpha+\alpha^{\prime}\right|} \\
& \quad \leq C^{k}|p|_{k}^{m, m^{\prime}}\langle\xi\rangle^{\tau_{1}}\langle x\rangle^{\tau_{2}}\left(1+\langle x\rangle^{2 \delta_{2}}|\eta|^{2}\right)^{-\ell}\langle\eta\rangle^{\left|m_{1}\right|+\rho_{1}|\alpha|},
\end{aligned}
$$

where $k=\left|\alpha+\beta+\alpha^{\prime}+\beta^{\prime}\right|+2 \ell$. Therefore, for $2 \ell>\left|m_{1}\right|+\rho_{1}|\alpha|+n$,

$$
\begin{aligned}
\left|I_{1}\right| & =\left|\int_{\mathbb{R}_{\eta}^{n}} \int_{|y| \leq 4^{-1}\langle x\rangle^{\delta_{2}}} e^{-i y \eta} r_{\theta}(x, \xi ; \eta, y) \chi(y) d y d \eta\right| \\
& \leq C^{k}|p|_{k}^{m, m^{\prime}}\langle\xi\rangle^{\tau_{1}}\langle x\rangle^{\tau_{2}} \int\left(1+\langle x\rangle^{2 \delta_{2}}|\eta|^{2}\right)^{-\ell}\langle\eta\rangle^{\left|m_{1}\right|+\rho_{1}|\alpha|} d \eta \int_{|y| \leq \frac{\left\langle x \delta_{2}\right.}{4}} d y \\
& \leq C^{k}|p|_{k}^{m, m^{\prime}}\langle\xi\rangle^{\tau_{1}}\langle x\rangle^{\tau_{2}} \int\langle\eta\rangle^{-2 \ell}\left\langle\langle x\rangle^{-\delta_{2}} \eta\right\rangle^{\left|m_{1}\right|+\rho_{1}|\alpha|} d \eta \int_{|y| \leq \frac{\left\langle x \delta_{2} \delta_{2}\right.}{4}\langle x\rangle^{-\delta_{2} n} d y} \\
& \leq C^{k}|p|_{k}^{m, m^{\prime}}\langle\xi\rangle^{\tau_{1}}\langle x\rangle^{\tau_{2}} \int\langle\eta\rangle^{-2 \ell+\left|m_{1}\right|+\rho_{1}|\alpha|} d \eta \prod_{j=1}^{n} \int_{\left|y_{j}\right| \leq \frac{\left\langle x \delta_{2}\right.}{4}\langle x\rangle^{-\delta_{2} n} d y_{j}} \\
& \leq C^{k} \int\langle\eta\rangle^{-n} \overline{d \eta}|p|_{k}^{m, m^{\prime}}\langle\xi\rangle^{\tau_{1}}\langle x\rangle^{\tau_{2}} .
\end{aligned}
$$

To estimate $I_{2}$ and $I_{3}$, it is useful to study $\left|\Delta_{\eta}^{\ell_{1}} r_{\theta}\right|$. We have

$$
\begin{aligned}
\left|\Delta_{\eta}^{\ell_{1}} r_{\theta}\right| & \leq \sum_{|Q|=\ell_{1}} \frac{\ell_{1} !}{Q !} \sum_{Q_{1}+Q_{2}=2 Q} \frac{(2 Q) !}{Q_{1} ! Q_{2} !}\left|\partial_{\eta}^{Q_{1}}\left(1+\langle x\rangle^{2 \delta_{2}}|\eta|^{2}\right)^{-\ell}\right| \cdot\left|\left(1-\langle x\rangle^{2 \delta_{2}} \Delta_{y}\right)^{\ell} \partial_{\eta}^{Q_{2}} q\right| \\
& \leq \sum_{|Q|=\ell_{1}} \frac{\ell_{1} !}{Q !} \sum_{Q_{1}+Q_{2}=2 Q} \frac{(2 Q) !}{Q_{1} ! Q_{2} !} C^{\left|Q_{1}\right|+\ell+1}\langle x\rangle^{\delta_{2}\left|Q_{1}\right|} Q_{1} !\left(1+\langle x\rangle^{2 \delta_{2}}|\eta|^{2}\right)^{-\ell-\left|Q_{1}\right|}|\theta|^{\left|Q_{2}\right|} \\
& \times\left|\left(1-\langle x\rangle^{2 \delta_{2}} \Delta_{y}\right)^{\ell} p_{\left(\beta, \beta^{\prime}\right)}^{\left(\alpha+Q_{2}, \alpha^{\prime}\right)}\right| .
\end{aligned}
$$

Noticing that

$$
\begin{aligned}
\left|\left(1-\langle x\rangle^{2 \delta_{2}} \Delta_{y}\right)^{\ell} p_{\left(\beta, \beta^{\prime}\right)}^{\left(\alpha+Q_{2}, \alpha^{\prime}\right)}\right| & \leq \sum_{\ell_{0}+|L|=\ell} \frac{\ell !}{\ell_{0} ! L !}\langle x\rangle^{2 \delta_{2}|L|}\left|p_{\left(\beta, \beta^{\prime}+2 L\right)}^{\left(\alpha+Q_{2}, \alpha^{\prime}\right)}\right| \\
& \leq \sum_{\ell_{0}+|L|=\ell} \frac{\ell !}{\ell_{0} ! L !}\langle x\rangle^{2 \delta_{2}|L|}|p|_{\tilde{k}}^{m, m^{\prime}}\langle\xi+\theta \eta\rangle^{m_{1}-\rho_{1}\left|\alpha+Q_{2}\right|}\langle\xi\rangle^{m_{1}^{\prime}-\rho_{1}\left|\alpha^{\prime}\right|} \\
& \times\langle x\rangle^{m_{2}-\rho_{2}|\beta|}\langle x+y\rangle^{m_{2}^{\prime}-\rho_{2}\left|\beta^{\prime}+2 L\right|}\langle x ; x+y\rangle^{\delta_{2}\left|\alpha+\alpha^{\prime}+Q_{2}\right|},
\end{aligned}
$$

where $\tilde{k}=\left|\alpha+\alpha^{\prime}+\beta+\beta^{\prime}\right|+2\left(\ell_{1}+\ell\right)$, we obtain

$$
\begin{aligned}
\left|\Delta_{\eta}^{\ell_{1}} r_{\theta}\right| \leq \sum_{|Q|=\ell_{1}} \frac{\ell_{1} !}{Q !} \sum_{Q_{1}+Q_{2}=2 Q} \frac{(2 Q) !}{Q_{1} ! Q_{2} !} C^{\left|Q_{1}\right|+\ell+1}\langle x\rangle^{\delta_{2}\left|Q_{1}\right|} Q_{1} ! \\
\quad \times\left(1+\langle x\rangle^{2 \delta_{2}}|\eta|^{2}\right)^{-\ell-\left|Q_{1}\right|} \sum_{\ell_{0}+|L|=\ell} \frac{\ell !}{\ell_{0} ! L !}\langle x\rangle^{2 \delta_{2}|L|}|p|_{\tilde{k}}^{m, m^{\prime}}\langle\xi+\theta \eta\rangle^{m_{1}-\rho_{1}\left|\alpha+Q_{2}\right|} \\
\quad \times\langle\xi\rangle^{m_{1}^{\prime}-\rho_{1}\left|\alpha^{\prime}\right|}\langle x\rangle^{m_{2}-\rho_{2}|\beta|}\langle x+y\rangle^{m_{2}^{\prime}-\rho_{2}\left|\beta^{\prime}+2 L\right|}\langle x ; x+y\rangle^{\delta_{2}\left|\alpha+\alpha^{\prime}+Q_{2}\right|} .
\end{aligned}
$$


Now, we proceed with the estimate for $I_{2}$. If $|y| \leq 2^{-1}\langle x\rangle$, we get

$$
\left|\Delta_{\eta}^{\ell} r_{\theta}\right| \leq C^{\tilde{k}+1}|p|_{\tilde{k}}^{m, m^{\prime}}\langle\xi\rangle^{\tau_{1}}\langle x\rangle^{\tau_{2}+2 \delta_{2} \ell}\left(1+\langle x\rangle^{2 \delta_{2}}|\eta|^{2}\right)^{-\ell}\langle\eta\rangle^{\left|m_{1}\right|+\rho_{1}|\alpha|},
$$

therefore, using integration by parts and assuming $2 \ell>\left|m_{1}\right|+\rho_{1}|\alpha|+2 n$,

$$
\left|I_{2}\right| \leq C^{\tilde{k}+1}|p|_{\tilde{k}}^{m, m^{\prime}}\langle\tilde{\zeta}\rangle^{\tau_{1}}\langle x\rangle^{\tau_{2}+\delta_{2}(2 \ell-n)} \int_{\frac{\left\langle x \delta_{2}\right.}{4} \leq|y| \leq \frac{\langle x\rangle}{2}}|y|^{-2 \ell} d y .
$$

For $|y| \geq 4^{-1}\langle x\rangle^{\delta_{2}}$, we may write

$$
|y|^{-2 \ell} \leq 2^{2 \ell} \prod_{j=1}^{n}\left(\left|y_{j}\right|+\frac{\langle x\rangle^{\delta_{2}}}{4}\right)^{-\frac{2 \ell}{n}},
$$

and then

$$
\int_{\frac{\langle x\rangle^{\delta_{2}}}{4} \leq|y| \leq \frac{\langle x\rangle}{2}}|y|^{-2 \ell} d y \leq 2^{2 \ell} \prod_{j=1}^{n} \int\left(\left|y_{j}\right|+\frac{\langle x\rangle^{\delta_{2}}}{4}\right)^{-\frac{2 \ell}{n}} d y_{j} \leq C^{\ell}\langle x\rangle^{\delta_{2}(n-2 \ell)} .
$$

After that,

$$
\left|I_{2}\right| \leq \tilde{C}^{\tilde{k}+1}|p|_{\tilde{k}}^{m, m^{\prime}}\langle\tilde{\xi}\rangle^{\tau_{1}}\langle x\rangle^{\tau_{2}}
$$

Finally, we take care of $I_{3}$. If $|y| \geq 4^{-1}\langle x\rangle$, we have $\langle x+y\rangle \leq 5|y|$ and $\langle x ; x+y\rangle \leq 9|y|$. Hence, for $|y| \geq 4^{-1}\langle x\rangle$, we may write

$$
\left|\Delta_{\eta}^{\ell_{1}} r_{\theta}\right| \leq C^{\tilde{k}+1}|p|_{\tilde{k}}^{m, m^{\prime}}\langle\xi\rangle^{\tau_{1}}\langle\eta\rangle^{\left|m_{1}\right|+\rho_{1}|\alpha|}\langle x\rangle^{m_{2}-\rho_{2}|\beta|}|y|^{\left|m_{2}^{\prime}\right|+\delta_{2}\left|\alpha+\alpha^{\prime}\right|+2 \delta_{2}\left(\ell+\ell_{1}\right)}
$$

and therefore, choosing $\ell, \ell_{1} \in \mathbb{N}_{0}$ satisfying $2 \ell>\left|m_{1}\right|+\rho_{1}|\alpha|+2 n$ and $2 \ell_{1}\left(1-\delta_{2}\right) \geq\left|m_{2}^{\prime}\right|+\delta_{2} \mid \alpha+$ $\alpha^{\prime} \mid+2 \delta_{2} \ell+2 n$,

$$
\begin{aligned}
\left|I_{3}\right| & \leq C^{\tilde{k}+1}|p|_{\tilde{k}}^{m, m^{\prime}}\langle\xi\rangle^{\tau_{1}}\langle x\rangle^{m_{2}-\rho_{2}|\beta|-\delta_{2} n} \int\langle\eta\rangle^{\left|m_{1}\right|+\rho_{1}|\alpha|-2 \ell} d \eta \\
& \times \int_{|y| \geq 4^{-1}\langle x\rangle}|y|^{\left|m_{2}^{\prime}\right|+\delta_{2}\left|\alpha+\alpha^{\prime}\right|+2 \delta_{2} \ell-2 \ell_{1}\left(1-\delta_{2}\right)} d y .
\end{aligned}
$$

Setting $r=2 \ell_{1}\left(1-\delta_{2}\right)-\left|m_{2}^{\prime}\right|-\delta_{2}\left|\alpha+\alpha^{\prime}\right|-2 \delta_{2} \ell$, we obtain

$$
\left|I_{3}\right| \leq C^{\tilde{k}+1}|p|_{\tilde{k}}^{m, m^{\prime}}\langle\xi\rangle^{\tau_{1}}\langle x\rangle^{m_{2}-\rho_{2}|\beta|} \int\langle\eta\rangle^{-2 n} \tilde{d \eta}\langle x\rangle^{n\left(1-\delta_{2}\right)-r} .
$$

Choosing $\ell_{1}$ such that $r>-m_{2}+\rho_{2}\left|\beta^{\prime}\right|-\delta_{2}\left|\alpha+\alpha^{\prime}\right|+n\left(1-\delta_{2}\right)$, we get

$$
\left|I_{3}\right| \leq C^{\tilde{k}+1}|p|_{\tilde{k}}^{m, m^{\prime}}\langle\tilde{\zeta}\rangle^{\tau_{1}}\langle x\rangle^{\tau_{2}} \int\langle\eta\rangle^{-2 n} \tilde{d \eta} .
$$

Gathering all the previous computations and choosing $\ell, \ell_{1} \in \mathbb{N}_{0}$ satisfying $2 \ell \geq\left|m_{1}\right|+\rho_{1}|\alpha|+2 n$,

$$
2 \ell_{1}\left(1-\delta_{2}\right) \geq 2\left|m_{2}^{\prime}\right|+\rho_{2}\left|\beta^{\prime}\right|+\delta_{2}\left|\alpha+\alpha^{\prime}\right|+2 \delta_{2} \ell+2 n,
$$

we have

$$
\left|q_{\theta}(x, \xi)\right| \leq C^{\tilde{k}}|p|_{\tilde{k}}^{m, m^{\prime}}\langle\xi\rangle^{\tau_{1}}\langle x\rangle^{\tau_{2}},
$$

where $\tilde{k}=\left|\alpha+\beta+\alpha^{\prime}+\beta^{\prime}\right|+2\left(\ell+\ell_{1}\right)$. This concludes the proof. 
Remark 4. Let $a \in C^{\infty}\left(\mathbb{R}^{n}\right)$ such that $\left|\partial_{x}^{\beta} a(x)\right| \leq C_{\beta}\langle x\rangle^{m_{2}-|\beta|}$, for $\beta \in \mathbb{N}_{0}^{n}$. For each fixed $x$, we can look at $a(x+\cdot)$ as an amplitude in $\mathcal{A}_{(0,0)}^{\left(0,\left|m_{2}\right|\right)}\left(\mathbb{R}^{2 n}\right)$ and, for $\chi \in \mathscr{S}\left(\mathbb{R}^{n}\right), \chi(0)=1$,

$$
\begin{aligned}
\text { Os }-\left[e^{-i \eta y} a(x+y)\right] & =O s-\left[e^{-i \eta(y-x)} a(y)\right] \\
& =\lim _{\varepsilon \rightarrow 0} \iint e^{-i \eta(y-x)} \chi(\varepsilon \eta) \chi(\varepsilon y) a(y) d y d \eta \\
& =\lim _{\varepsilon \rightarrow 0} \int a(y) \chi(\varepsilon y) \varepsilon^{-n} \mathcal{F}^{-1}(\chi)\left(\varepsilon^{-1}(x-y)\right) d y \\
& =\lim _{\varepsilon \rightarrow 0} \int a(x-\varepsilon y) \chi(\varepsilon(x-\varepsilon y)) \mathcal{F}^{-1}(\chi)(y) d y \\
& =a(x) \int \mathcal{F}^{-1} \chi(y) d y=a(x) .
\end{aligned}
$$

Theorem 2. Let $p\left(x, \xi, x^{\prime}, \xi^{\prime}\right) \in S G_{\rho,\left(0, \delta_{2}\right)}^{m, m^{\prime}}$ and set

$$
p_{L}(x, \xi)=O s-\iint e^{-i \eta y} p(x, \xi+\eta, x+y, \xi) d y d \eta, \quad x, \xi \in \mathbb{R}^{n} .
$$

Then, $p_{L} \in S G_{\rho,\left(0, \delta_{2}\right)^{\prime}}^{m+m^{\prime}} p\left(x, D_{x}, x^{\prime}, D_{x^{\prime}}\right)=p_{L}\left(x, D_{x}\right)$ and

$$
p_{L}(x, \xi) \sim \sum_{j \in \mathbb{N}_{0}} \sum_{|\alpha|=j} \frac{1}{\alpha !}\left(\partial_{\xi}^{\alpha} D_{x^{\prime}}^{\alpha} p\right)(x, \xi, x, \xi)
$$

Furthermore, given $\ell \in \mathbb{N}_{0}$, there is $\ell_{0}:=\ell_{0}(\ell) \in \mathbb{N}_{0}$ such that

$$
\left|p_{L}\right|_{\ell}^{m+m^{\prime}} \leq C_{\ell, \ell_{0}}|p|_{\ell_{0}}^{\left(m, m^{\prime}\right)}
$$

Proof. First, we notice that, repeating the ideas in the proof of [5] (Lemma 2.3, Page 65), we can conclude that $p_{L}=p$ as operators.

Applying Lemma 1 for $\alpha=\alpha^{\prime}=\beta^{\prime}=\beta=0$, we obtain that $p_{L} \in \mathbf{S G}_{\rho,\left(0, \delta_{2}\right)}^{m+m^{\prime}}$.

Now, by Taylor formula, we may write

$$
\begin{aligned}
p(x, \xi+\eta, & x+y, \xi)=\sum_{|\alpha|<N} \frac{\eta^{\alpha}}{\alpha !}\left(\partial_{\xi}^{\alpha} p\right)(x, \xi, x+y, \xi) \\
& +N \sum_{|\gamma|=N} \frac{\eta^{\gamma}}{\gamma !} \int_{0}^{1}(1-\theta)^{N-1}\left(\partial_{\xi}^{\gamma} p\right)(x, \xi+\theta \eta, x+y, \xi) d \theta .
\end{aligned}
$$

Integration by parts and Remark 4 give

$$
\begin{aligned}
O s-\left[e^{-i \eta y} \eta^{\alpha}\left(\partial_{\xi}^{\alpha} p\right)(x, \xi, x+y, \xi)\right] & =O s-\left[e^{-i \eta y} D_{y}^{\alpha}\left(\partial_{\xi}^{\alpha} p\right)(x, \xi, x+y, \xi)\right] \\
& =\left(\partial_{\xi}^{\alpha} D_{x^{\prime}}^{\alpha} p\right)(x, \xi, x, \xi)
\end{aligned}
$$

and

$$
\begin{aligned}
& \text { Os- }\left[e^{-i \eta y} \eta^{\gamma} \int_{0}^{1}(1-\theta)^{N-1}\left(\partial_{\xi}^{\gamma} p\right)(x, \xi+\theta \eta, x+y, \xi) d \theta\right]= \\
& \text { Os }-\left[e^{-i \eta y} \int_{0}^{1}(1-\theta)^{N-1}\left(\partial_{\xi}^{\gamma} D_{x^{\prime}}^{\gamma} p\right)(x, \xi+\theta \eta, x+y, \xi) d \theta\right] .
\end{aligned}
$$

Hence

$$
p_{L}(x, \xi)=\sum_{|\alpha|<N} \frac{1}{\alpha !}\left(\partial_{\xi}^{\alpha} D_{x^{\prime}}^{\alpha} p\right)(x, \xi, x, \xi)+r_{N}(x, \xi)
$$


and Lemma 1 implies $r_{N} \in \mathbf{S G}_{\rho,\left(0, \delta_{2}\right)}^{m+m^{\prime}-N\left(\rho-\left(0, \delta_{2}\right)\right)}$.

To obtain the same kind of result for the classes $\mathbf{S G}_{\rho,\left(0, \delta_{2}\right) ;(\mu, v)}^{m, m^{\prime}}$, we need an analog of Lemma 1 with a precise estimate of the Gevrey regularity.

Lemma 2. Let $p \in S G_{\rho,\left(0, \delta_{2}\right) ;(\mu, v)}^{m, m^{\prime}}\left(\mathbb{R}^{4 n} ; A\right)$ for some $A>0$. For any multi-indices $\alpha, \alpha^{\prime}, \beta, \beta^{\prime}$ set $q=p_{\beta, \beta^{\prime}}^{\alpha, \alpha^{\prime}}$ and for $\theta \in[-1,1]$, consider $q_{\theta}$ as in Lemma 1. Then,

$$
\left|\partial_{\tilde{\xi}}^{\sigma} \partial_{x}^{\gamma} q_{\theta}(x, \xi)\right| \leq|p|_{A}^{m, m^{\prime}}\left(C A^{r}\right)^{k}\left(\alpha ! \alpha^{\prime} ! \sigma !\right)^{\tilde{\mu}}\left(\beta ! \beta^{\prime} ! \gamma !\right)^{\tilde{v}}\langle x\rangle^{\tau_{2}-\rho_{2}|\gamma|+\delta_{2}|\sigma|}\langle\xi\rangle^{\tau_{1}-\rho_{1}|\sigma|}
$$

where $k=\left|\alpha+\beta+\alpha^{\prime}+\beta^{\prime}+\sigma+\gamma\right|, \tilde{\mu}=\left(1+\frac{\rho_{1}+\delta_{2}}{1-\delta_{2}}\right) \mu+\rho_{1} v, \tilde{v}=\frac{\rho_{2}}{1-\delta_{2}} \mu+v, \tau_{1}$ and $\tau_{2}$ are as in Lemma 1 and $C, r$ are positive constants depending only on $\rho, \delta, m, m^{\prime}, \mu, v$ and $n$.

Proof. Following the ideas presented in the proof of Lemma 1 and using standard factorial inequalities, we obtain

$$
\left|q_{\theta}(x, \xi)\right| \leq|p|_{A}^{m, m^{\prime}}(C A)^{\tilde{k}} \ell !^{2 v} \ell_{1} !^{2 \mu}\left(\alpha ! \alpha^{\prime} !\right)^{\mu}\left(\beta ! \beta^{\prime} !\right)^{v}\langle\xi\rangle^{\tau_{1}}\langle x\rangle^{\tau_{2}},
$$

where $C>0$ depends only of $\mu, v, n, m_{1}, \tilde{k}=\left|\alpha+\beta+\alpha^{\prime}+\beta^{\prime}\right|+2\left(\ell+\ell_{1}\right)$ and $\ell, \ell_{1}$ are positive integers satisfying $2 \ell \geq\left|m_{1}\right|+\rho_{1}|\alpha|+2 n$, and

$$
2 \ell_{1}\left(1-\delta_{2}\right) \geq 2\left|m_{2}^{\prime}\right|+\rho_{2}\left|\beta^{\prime}\right|+\delta_{2}\left|\alpha+\alpha^{\prime}\right|+2 \delta_{2} \ell+2 n .
$$

In particular, if we choose

$$
\begin{gathered}
\ell=\left\lfloor\frac{\left|m_{1}\right|}{2}+\frac{\rho_{1}}{2}|\alpha|\right\rfloor+n+1, \\
\ell_{1}=\left\lfloor\frac{1}{1-\delta_{2}}\left(\left|m_{2}^{\prime}\right|+\delta_{2} \ell+\frac{\rho_{2}}{2}\left|\beta^{\prime}\right|+\frac{\delta_{2}}{2}\left|\alpha+\alpha^{\prime}\right|\right)\right\rfloor+n+1,
\end{gathered}
$$

where $\lfloor\cdot\rfloor$ stands for the floor function, then we obtain

$$
\left|q_{\theta}(x, \xi)\right| \leq|p|_{A}^{m, m^{\prime}}\left(C A^{r}\right)^{\left|\alpha+\beta+\alpha^{\prime}+\beta^{\prime}\right|} \alpha !^{\tilde{\mu}} \alpha^{\prime} !^{\mu\left(1+\frac{\delta_{2}}{1-\delta_{2}}\right)} \beta !^{v} \beta^{\prime} !^{\tilde{y}}\left(\beta ! \beta^{\prime} !\right)^{v}\langle\xi\rangle^{\tau_{1}}\langle x\rangle^{\tau_{2}} .
$$

From the last estimate and (10), we get (12).

As a consequence of Lemma 2, we have the following result.

Theorem 3. Let $p \in S G_{\rho,\left(0, \delta_{2}\right) ;(\mu, v)}^{m, m^{\prime}}\left(\mathbb{R}^{4 n}\right)$. Then, $p_{L}$ belongs to $S G_{\rho,\left(0, \delta_{2}\right) ;(\tilde{\mu}, \tilde{v})}^{m+m^{\prime}}$ and

$$
p_{L}(x, \xi) \sim \sum_{j \in \mathbb{N}_{0}^{n}} p_{j}(x, \xi) \quad \text { in } \quad F S G_{\rho,\left(0, \delta_{2}\right) ;(\tilde{\mu}, \tilde{v})^{\prime}}^{m+m^{\prime}}
$$

where

$$
p_{j}(x, \xi)=\sum_{|\alpha|=j} \alpha !^{-1}\left(\partial_{\xi}^{\alpha} D_{x^{\prime}}^{\alpha} p\right)(x, \xi, x, \xi)
$$

and $\tilde{\mu}$ and $\tilde{v}$ are as in Lemma 2.

Theorem 3 states that $p_{L}$ has a lower Gevrey regularity than $p$ since $\tilde{\mu}>\mu$ and $\tilde{v}>v$. However, we observe that, if $p \in \mathbf{S G}_{\rho,\left(0, \delta_{2}\right) ;(\mu, v)^{\prime}}^{m, m^{\prime}}$ then $\sum_{j \in \mathbb{N}_{0}} p_{j} \in F S G_{\rho,\left(0, \delta_{2}\right) ;(\mu, v)}^{m+m^{\prime}}$. Thus, by Proposition 1 , there exists $q \in \mathbf{S G}_{\rho,\left(0, \delta_{2}\right) ;(\mu, v)}^{m+m^{\prime}}$ such that $q \sim \sum p_{j}$ in $F S G_{\rho,\left(0, \delta_{2}\right) ;(\mu, v)}^{m+m^{\prime}}$. On the other hand, we have $p_{L} \sim \sum p_{j}$ in $\mathbf{F S}_{\rho,\left(0, \delta_{2}\right) ;(\tilde{\mu}, \tilde{v})}^{m+m^{\prime}}$. Hence, $p_{L}-q \sim 0$ in $F S G_{\rho,\left(0, \delta_{2}\right) ;(\tilde{\mu}, \tilde{v})}^{m+m^{\prime}}$, which implies that $p_{L}=q+r$, where $r$ belongs to the Gelfand-Shilov space $\mathcal{S}_{\tilde{\mu}+\tilde{v}-1}\left(\mathbb{R}^{2 n}\right)$. This means that we can write $p_{L}$ as the sum of a 
symbol with the same orders and regularity as $p$ plus a remainder term which has a lower Gevrey regularity but with orders small as we want. This is a crucial in the applications to the Cauchy problem for $p$-evolution equations because in the energy estimates the remainder terms can be neglected and does not affect the regularity of the solution (cf. [12]).

\section{The Friedrichs Part}

Fix $q \in C_{0}^{\infty}\left(\mathbb{R}^{n} ; \mathbb{R}\right)$ supported on $Q=\left\{\sigma \in \mathbb{R}^{n}:|\sigma| \leq 1\right\}$, such that $q$ is even, $\int q(\sigma)^{2} d \sigma=1$ and $\left|\partial_{\sigma}^{\alpha} q(\sigma)\right| \leq C_{q}^{|\alpha|+1} \alpha !^{s}$, where $1<s \leq \min \{\mu, v\}$. In this section, we consider $\mu, v>1$.

Lemma 3. For $\tau, \tau^{\prime} \in(0,1)$, set $F: \mathbb{R}^{3 n} \rightarrow \mathbb{R}$ given by

$$
F(x, \xi, \zeta)=q\left(\langle x\rangle^{\tau^{\prime}}\langle\xi\rangle^{-\tau}(\zeta-\xi)\right)\langle\xi\rangle^{-\frac{\tau n}{2}}\langle x\rangle^{\frac{\tau^{\prime} n}{2}}
$$

for $x, \xi, \zeta \in \mathbb{R}^{n}$. Then, for any $\alpha, \beta \in \mathbb{N}_{0}^{n}$, we have

$$
\begin{aligned}
\partial_{\xi}^{\alpha} \partial_{x}^{\beta} F(x, \xi, \zeta)= & \langle x\rangle^{\frac{\tau^{\prime} n}{2}}\langle\xi\rangle^{-\frac{\tau n}{2}} \sum_{\substack{|\gamma| \leq|\alpha| \\
\gamma_{1} \leq \gamma}} \sum_{\substack{\delta|\leq| \beta \mid\\
}} \psi_{\alpha \gamma \gamma_{1}}(\xi) \phi_{\beta \delta \gamma \gamma_{1}}(x) \\
& \times\left(\langle x\rangle^{\tau^{\prime}}\langle\xi\rangle^{-\tau}(\zeta-\xi)\right)^{\gamma_{1}+\delta}\left(\partial^{\gamma+\delta} q\right)\left(\langle x\rangle^{\tau^{\prime}}\langle\xi\rangle^{-\tau}(\zeta-\xi)\right),
\end{aligned}
$$

where $\psi_{\alpha \gamma \gamma_{1}}$ and $\phi_{\beta \delta \gamma \gamma_{1}}$ satisfy the following estimates:

$$
\begin{gathered}
\left|\partial_{\xi}^{\mu} \psi_{\alpha \gamma \gamma_{1}}(\xi)\right| \leq C_{\alpha \mu}\langle\xi\rangle^{-|\alpha|+(1-\tau)\left|\gamma-\gamma_{1}\right|-|\mu|}, \\
\left|\partial_{x}^{v} \phi_{\beta \delta \gamma \gamma_{1}}(x)\right| \leq C_{\beta v}\langle x\rangle^{-|\beta|+\tau^{\prime}\left|\gamma-\gamma_{1}\right|-|v|},
\end{gathered}
$$

for every $\mu, v \in \mathbb{N}_{0}^{n}$.

The lemma can be proved by induction on $|\alpha+\beta|$ following the same argument as in the proof of [5] [Lemma 4.1 page 129]. Observing that $\left|\gamma-\gamma_{1}\right| \leq|\gamma| \leq|\alpha|$ we have $\psi_{\alpha \gamma \gamma_{1}}(\xi) \phi_{\beta \delta \gamma \gamma_{1}}(x) \in$ SG $^{\left(-\tau|\alpha|,-|\beta|+\tau^{\prime}|\alpha|\right)}\left(\mathbb{R}^{2 n}\right)$. Finally, we remark that, for $\alpha=\beta=0$, we have $\psi_{\alpha \gamma \gamma_{1}} \equiv \phi_{\beta \delta \gamma \gamma_{1}} \equiv 1$.

Definition 11. Let $p \in S G^{m}$. Moreover, let $F(x, \xi, \zeta)$ be defined by (13) with $\tau=\tau^{\prime}=\frac{1}{2}$. We define the Friedrichs part of $p$ by

$$
p_{F}\left(\xi, x^{\prime}, \xi^{\prime}\right)=\int F\left(x^{\prime}, \xi, \zeta\right) p\left(x^{\prime}, \zeta\right) F\left(x^{\prime}, \xi^{\prime}, \zeta\right) d \zeta, \quad x^{\prime}, \xi^{\prime}, \xi^{\prime} \in \mathbb{R}^{n}
$$

The following properties can be proved as in [5]. We leave the details to the reader.

Proposition 6. Let $p \in S G^{m}$ and let $p_{F}$ be its Friedrichs part. For $u, v \in \mathscr{S}\left(\mathbb{R}^{n}\right)$, the following conditions hold:

(i) If $p(x, \xi)$ is real, then $\left(p_{F} u, v\right)_{L^{2}}=\left(u, p_{F} v\right)_{L^{2}}$.

(ii) If $p(x, \xi) \geq 0$, then $\left(p_{F} u, u\right)_{L^{2}} \geq 0$.

(iii) If $p(x, \xi)$ is purely imaginary, then $\left(p_{F} u, v\right)_{L^{2}}=-\left(u, p_{F} v\right)_{L^{2}}$.

(iv) If Re $p(x, \xi) \geq 0$, then $\operatorname{Re}\left(p_{F} u, u\right)_{L^{2}} \geq 0$.

Theorem 4. Let $p \in S G^{m}\left(\mathbb{R}^{2 n}\right)$ and let $p_{F}$ be its Friedrichs part. Then, $p_{F, L} \in S G^{m}\left(\mathbb{R}^{2 n}\right)$ and $p_{F, L}-p \in$ $S G^{m-(1,1)}\left(\mathbb{R}^{2 n}\right)$. Moreover,

$$
p_{F, L}(x, \xi)-p(x, \xi) \sim \sum_{|\beta|=1} q_{0, \beta}(x, \xi)+\sum_{|\alpha+\beta| \geq 2} q_{\alpha, \beta}(x, \xi)
$$


where, for $|\beta|=1$,

$$
\begin{aligned}
q_{0, \beta}(x, \xi) & =\sum_{\beta_{1}+\beta_{2}+\beta_{3}=\beta} D_{x}^{\beta_{3}} p(x, \xi) \sum_{|\gamma| \leq|\beta||\delta| \leq\left|\beta_{1}\right|} \psi_{\beta \gamma \gamma}(\xi) \phi_{\beta_{1} \delta \gamma \gamma}(x) \\
& \times \sum_{\left|\delta^{\prime}\right| \leq\left|\beta_{2}\right|} \phi_{\beta_{2} \delta^{\prime} 00}(x) \int \sigma^{\gamma+\delta+\delta^{\prime}}\left(\partial^{\gamma+\delta} q\right)(\sigma)\left(\partial^{\delta^{\prime}} q\right)(\sigma) d \sigma
\end{aligned}
$$

with $\psi_{\beta \gamma \gamma} \phi_{\beta_{1} \delta \gamma \gamma} \in S G^{\left(-|\beta|,-\left|\beta_{1}\right|\right)}\left(\mathbb{R}^{2 n}\right), \phi_{\beta_{2} \delta^{\prime} 00} \in S G^{\left(0,-\left|\beta_{2}\right|\right)}\left(\mathbb{R}^{2 n}\right)$ and, for $|\alpha+\beta| \geq 2$ :

$$
\begin{aligned}
q_{\alpha, \beta}(x, \xi) & =\sum_{\beta_{1}+\beta_{2}+\beta_{3}=\beta} \frac{\left(\langle\xi\rangle^{\frac{1}{2}}\langle x\rangle^{-\frac{1}{2}}\right)^{|\alpha|}}{\alpha ! \beta_{1} ! \beta_{2} ! \beta_{3} !} \\
& \times \sum_{\substack{|\gamma| \leq|\beta| \\
\gamma 1 \leq \gamma}} \sum_{\beta \gamma|\leq| \beta_{1} \mid} \psi_{\beta \gamma \gamma_{1}}(\xi) \phi_{\beta_{1} \delta \gamma \gamma_{1}}(x) \sum_{\left|\delta^{\prime}\right| \leq\left|\beta_{2}\right|} \phi_{\beta_{2} \delta^{\prime} 00}(x) \\
& \times \int_{Q} \sigma^{\alpha+\gamma_{1}+\delta_{1}+\delta_{1}^{\prime}}\left(\partial^{\gamma+\delta} q\right)(\sigma)\left(\partial^{\delta^{\prime}} q\right)(\sigma) d \sigma \cdot \partial_{\tilde{\xi}}^{\alpha} D_{x}^{\beta_{3}} p(x, \xi)
\end{aligned}
$$

with $\psi_{\beta \gamma \gamma_{1}} \phi_{\beta_{1} \delta \gamma \gamma_{1}} \in S G^{\left(-\frac{1}{2}|\beta|,-\left|\beta_{1}\right|+\frac{1}{2}|\beta|\right)}\left(\mathbb{R}^{2 n}\right), \phi_{\beta_{2} \delta^{\prime} 00} \in S G^{\left(0,-\left|\beta_{2}\right|\right)}\left(\mathbb{R}^{2 n}\right)$.

We need the following technical lemma whose proof follows by a compactness argument.

Lemma 4. For $\tau \in(0,1)$, there is $C>0$ such that

$$
C^{-1}\langle\xi\rangle \leq\left\langle\xi+\zeta\langle\xi\rangle^{\tau}\right\rangle \leq C\langle\xi\rangle
$$

for every $\xi \in \mathbb{R}^{n}$ and $|\zeta| \leq 1$.

Proof of Theorem 4. From Leibniz formula and Cauchy-Schwartz inequality, we get

$$
\begin{aligned}
& \left|\partial_{\xi}^{\alpha} \partial_{\xi^{\prime}}^{\alpha^{\prime}} \partial_{x^{\prime}}^{\beta^{\prime}} p_{F}\left(\xi, x^{\prime}, \xi^{\prime}\right)\right| \\
& \quad \leq \sum_{\beta_{1}^{\prime}+\beta_{2}^{\prime}+\beta_{3}^{\prime}=\beta^{\prime}} \frac{\beta^{\prime} !}{\beta_{1}^{\prime} ! \beta_{2}^{\prime} ! \beta_{3}^{\prime} !}\left[\int\left|\partial_{\xi}^{\alpha} \partial_{x^{\prime}}^{\beta_{1}^{\prime}} F\left(x^{\prime}, \xi, \zeta\right)\right|^{2} d \zeta\right]^{\frac{1}{2}} \cdot\left[\int\left|\partial_{x^{\prime}}^{\beta_{2}^{\prime}} p\left(x^{\prime}, \zeta\right) \partial_{\xi^{\prime}}^{\alpha^{\prime}} \beta_{x^{\prime}}^{\beta_{3}^{\prime}} F\left(x^{\prime}, \xi^{\prime}, \zeta\right)\right|^{2} d \zeta\right]^{\frac{1}{2}} .
\end{aligned}
$$

Now, by changing variables, we obtain

$$
\begin{aligned}
& \left|\partial_{\xi}^{\alpha} \partial_{\xi^{\prime}}^{\alpha^{\prime}} \partial_{x^{\prime}}^{\beta^{\prime}} p_{F}\left(\xi, x^{\prime}, \xi^{\prime}\right)\right| \\
& \leq\langle\xi\rangle^{\frac{n}{4}}\left\langle x^{\prime}\right\rangle^{-\frac{n}{2}}\left\langle\xi^{\prime}\right\rangle^{\frac{n}{4}} \sum_{\beta_{1}^{\prime}+\beta_{2}^{\prime}+\beta_{3}^{\prime}=\beta^{\prime}} \frac{\beta_{1}^{\prime} !}{\beta_{1}^{\prime} ! \beta_{2}^{\prime} ! \beta_{3}^{\prime} !}\left[\int_{Q}\left|\left(\partial_{\xi}^{\alpha} \partial_{x^{\prime}}^{\beta_{1}^{\prime}} F\right)\left(x^{\prime}, \xi,\left\langle x^{\prime}\right\rangle^{-\frac{1}{2}}\langle\xi\rangle^{\frac{1}{2}} \sigma+\xi\right)\right|^{2} d \sigma\right]^{\frac{1}{2}} \\
& \times\left[\int_{Q}\left|\left(\partial_{\xi^{\prime}}^{\alpha^{\prime}} \beta_{x^{\prime}}^{\beta_{2}^{\prime}} F\right)\left(x^{\prime}, \xi^{\prime},\left\langle x^{\prime}\right\rangle^{-\frac{1}{2}}\left\langle\xi^{\prime}\right\rangle^{\frac{1}{2}} \sigma+\xi^{\prime}\right)\left(\partial_{x^{\prime}}^{\beta_{3}^{\prime}} p\right)\left(x^{\prime},\left\langle x^{\prime}\right\rangle^{-\frac{1}{2}}\left\langle\xi^{\prime}\right\rangle^{\frac{1}{2}} \sigma+\xi^{\prime}\right)\right|^{2} d \sigma\right]^{\frac{1}{2}} .
\end{aligned}
$$


Applying Lemma 3, we obtain

$$
\begin{aligned}
& \left|\partial_{\xi}^{\alpha} \partial_{\xi^{\prime}}^{\alpha^{\prime}} \partial_{x^{\prime}}^{\beta^{\prime}} p_{F}\left(\xi, x^{\prime}, \xi^{\prime}\right)\right| \\
& \leq \sum_{\beta_{1}^{\prime}+\beta_{2}^{\prime}+\beta_{3}^{\prime}=\beta^{\prime}} \frac{\beta^{\prime} !}{\beta_{1}^{\prime} ! \beta_{2}^{\prime} ! \beta_{3}^{\prime} !}\left[\int \sum_{\substack{|\gamma| \leq|\alpha||\delta| \leq\left|\beta_{1}^{\prime}\right| \\
\gamma 1 \leq \gamma}}\left|\psi_{\alpha \gamma \gamma_{1}}(\xi) \phi_{\beta_{1}^{\prime} \delta \gamma \gamma_{1}}\left(x^{\prime}\right) \sigma^{\gamma_{1}+\delta}\left(\partial^{\gamma+\delta} q\right)(\sigma)\right|^{2} d \sigma\right]^{\frac{1}{2}} \\
& \times\left[\int \sum_{\substack{|\gamma| \leq\left|\alpha^{\prime}\right| \delta|\leq| \beta_{2}^{\prime} \mid \\
\gamma 1 \leq \gamma}}\left|\psi_{\alpha^{\prime} \gamma \gamma_{1}}\left(\xi^{\prime}\right) \phi_{\beta_{2}^{\prime} \delta \gamma \gamma_{1}}\left(x^{\prime}\right) \sigma^{\gamma_{1}+\delta}\left(\partial^{\gamma+\delta} q\right)(\sigma)\right|^{2}\left|\partial_{x^{\prime}}^{\beta_{3}^{\prime}} p\left(x^{\prime},\left\langle x^{\prime}\right\rangle^{-\frac{1}{2}}\left\langle\xi^{\prime}\right\rangle^{\frac{1}{2}} \sigma+\xi^{\prime}\right)\right|^{2} d \sigma\right]^{\frac{1}{2}} \text {. }
\end{aligned}
$$

We now observe that by Lemma 4

$$
\left|\partial_{x^{\prime}}^{\beta_{3}^{\prime}} p\left(x^{\prime},\left\langle x^{\prime}\right\rangle^{-\frac{1}{2}}\left\langle\xi^{\prime}\right\rangle^{\frac{1}{2}} \sigma+\xi^{\prime}\right)\right| \leq C_{\beta_{3}^{\prime}}\left\langle\xi^{\prime}\right\rangle^{m_{1}}\left\langle x^{\prime}\right\rangle^{m_{2}-\left|\beta_{3}^{\prime}\right|} .
$$

Since $\psi_{\alpha \gamma \gamma_{1}} \phi_{\beta_{1}^{\prime} \delta \gamma \gamma_{1}} \in \mathbf{S G}^{\left(-\frac{|\alpha|}{2},-\left|\beta_{1}^{\prime}\right|+\frac{|\alpha|}{2}\right)}$ and $\psi_{\alpha^{\prime} \gamma \gamma_{1}} \phi_{\beta_{2}^{\prime} \delta \gamma \gamma_{1}} \in \mathbf{S G}^{\left(-\frac{\left|\alpha^{\prime}\right|}{2},-\left|\beta_{2}^{\prime}\right|+\frac{\left|\alpha^{\prime}\right|}{2}\right)}$ we obtain

$$
\left|\partial_{\xi}^{\alpha} \partial_{\xi^{\prime}}^{\alpha^{\prime}} \partial_{x^{\prime}}^{\beta^{\prime}} p_{F}\left(\xi, x^{\prime}, \xi^{\prime}\right)\right| \leq C_{\alpha \alpha^{\prime} \beta^{\prime}}\langle\xi\rangle^{-\frac{|\alpha|}{2}}\left\langle\xi^{\prime}\right\rangle^{m_{1}-\frac{\left|\alpha^{\prime}\right|}{2}}\left\langle x^{\prime}\right\rangle^{m_{2}-\left|\beta^{\prime}\right|+\frac{\left|\alpha+\alpha^{\prime}\right|}{2}},
$$

that is $p_{F} \in \mathbf{S G}_{(1 / 2,1),(0,1 / 2)}^{(0,0),\left(m_{1}, m_{2}\right)}$. Then, by Theorem $2, p_{F, L} \in \mathbf{S G}_{(1 / 2,1),(0,1 / 2)}^{m}$ and

$$
p_{F, L}(x, \xi) \sim \sum_{\beta} \frac{1}{\beta !}\left(\partial_{\xi}^{\beta} D_{x^{\prime}}^{\beta} p_{F}\right)(\xi, x, \xi)=\sum_{\beta} \tilde{p}_{\beta}(x, \xi),
$$

which implies that $p_{F, L}-\sum_{|\beta|<N} \tilde{p}_{\beta} \in \mathbf{S G}_{(1 / 2,1),(0,1 / 2)}^{m-\frac{N}{2}(1,1)}$ for every $N \in \mathbb{N}$. To improve this result, let us study more carefully the above asymptotic expansion. Note that

$$
\begin{aligned}
& \tilde{p}_{\beta}(x, \xi)=\sum_{\beta_{1}+\beta_{2}+\beta_{3}=\beta} \frac{1}{\beta_{1} ! \beta_{2} ! \beta_{3} !} \int \partial_{\xi}^{\beta} D_{x}^{\beta_{1}} F(x, \xi, \zeta) D_{x}^{\beta_{3}} p(x, \zeta) D_{x}^{\beta_{2}} F(x, \xi, \zeta) d \zeta \\
& =\sum_{\beta_{1}+\beta_{2}+\beta_{3}=\beta} \frac{1}{\beta_{1} ! \beta_{2} ! \beta_{3} !} \sum_{\substack{|\gamma| \leq|\beta| \\
\gamma 1 \leq \gamma}} \sum_{|\delta| \leq\left|\beta_{1}\right|} \psi_{\beta \gamma \gamma_{1}}(\xi) \phi_{\beta_{1} \delta \gamma \gamma_{1}}(x) \sum_{\left|\delta^{\prime}\right| \leq\left|\beta_{2}\right|} \psi_{\beta_{2} \delta^{\prime} 00}(x) \\
& \quad \times \int_{Q}\left(D_{x}^{\beta_{3}} p\right)\left(x,\langle\xi\rangle^{\frac{1}{2}}\langle x\rangle^{-\frac{1}{2}} \sigma+\xi\right) \sigma^{\gamma_{1}+\delta+\delta^{\prime}}\left(\partial^{\gamma+\delta} q\right)(\sigma)\left(\partial^{\delta^{\prime}} q\right)(\sigma) d \sigma .
\end{aligned}
$$

By Taylor formula, we can write

$$
\begin{aligned}
& D_{x}^{\beta_{3}} p\left(x,\langle\xi\rangle^{\frac{1}{2}}\langle x\rangle^{-\frac{1}{2}} \sigma+\xi\right)=\sum_{|\alpha|<N} \frac{\left(\langle\xi\rangle^{\frac{1}{2}}\langle x\rangle^{-\frac{1}{2}}\right)^{|\alpha|} \sigma^{\alpha}}{\alpha !} \partial_{\xi}^{\alpha} D_{x}^{\beta_{3}} p(x, \xi) \\
& \quad+N \sum_{|\alpha|=N} \frac{\langle\xi\rangle^{\frac{N}{2}}\langle x\rangle^{-\frac{N}{2}} \sigma^{\alpha}}{\alpha !} \int_{0}^{1}(1-\theta)^{N-1}\left(\partial_{\xi}^{\alpha} D_{x}^{\beta_{3}} p\right)\left(x, \theta\langle\xi\rangle^{\frac{1}{2}}\langle x\rangle^{-\frac{1}{2}} \sigma+\xi\right) d \theta
\end{aligned}
$$


Then, we get

$$
\begin{aligned}
\tilde{p}_{\beta}(x, \xi) & =\sum_{\substack{\beta_{1}+\beta_{2}+\beta_{3}=\beta|\alpha|<N\\
}} \frac{\left(\langle\xi\rangle^{\frac{1}{2}}\langle x\rangle^{-\frac{1}{2}}\right)^{|\alpha|}}{\alpha ! \beta_{1} ! \beta_{2} ! \beta_{3} !} \\
& \times \sum_{\substack{|\gamma| \leq|\beta||\delta| \leq\left|\beta_{1}\right| \\
\gamma_{1} \leq \gamma}} \psi_{\beta \gamma \gamma_{1}}(\xi) \phi_{\beta_{1} \delta \gamma \gamma_{1}}(x) \sum_{\left|\delta^{\prime}\right| \leq\left|\beta_{2}\right|} \phi_{\beta_{2} \delta^{\prime} 00}(x) \\
& \times \int_{Q} \sigma^{\alpha+\gamma_{1}+\delta+\delta^{\prime}} \partial^{\gamma+\delta} q(\sigma) \partial^{\delta^{\prime}} q(\sigma) d \sigma \cdot \partial_{\xi}^{\alpha} D_{x}^{\beta_{3}} p(x, \xi)+r_{\beta, N}(x, \xi)
\end{aligned}
$$

where

$$
\begin{aligned}
r_{\beta, N}(x, \xi)=\sum_{\beta_{1}+\beta_{2}+\beta_{3}=\beta} \sum_{|\alpha|=N} \frac{N}{\alpha !} \cdot \frac{\left(\langle\xi\rangle^{\frac{1}{2}}\langle x\rangle^{-\frac{1}{2}}\right)^{N}}{\beta_{1} ! \beta_{2} ! \beta_{3} !} \sum_{\substack{|\gamma| \leq|\beta| \\
\gamma 1 \leq \gamma|\leq| \beta_{1} \mid}} \psi_{\beta \gamma \gamma_{1}}(\xi) \phi_{\beta_{1} \delta \gamma \gamma_{1}}(x) \sum_{\left|\delta^{\prime}\right| \leq\left|\beta_{2}\right|} \phi_{\beta_{2} \delta^{\prime} 00}(x) \\
\quad \times \int_{Q} \sigma^{\gamma_{1}+\delta+\delta^{\prime}+\alpha} \partial^{\gamma+\delta} q(\sigma) \partial^{\delta^{\prime}} q(\sigma) \int_{0}^{1}(1-\theta)^{N-1}\left(\partial_{\xi}^{\alpha} D_{x}^{\beta_{3}} p\right)\left(x, \theta\langle\xi\rangle^{\frac{1}{2}}\langle x\rangle^{-\frac{1}{2}} \sigma+\xi\right) d \theta d \sigma .
\end{aligned}
$$

Using Lemma 4 , we get that $r_{\beta, N}$ belongs to $\mathbf{S G}^{\left(m_{1}-\frac{1}{2}(N+|\beta|), m_{2}-\frac{1}{2}(|\beta|+N)\right)}$, whereas

$$
\begin{aligned}
q_{\alpha, \beta}(x, \xi) & =\sum_{\substack{\beta_{1}+\beta_{2}+\beta_{3}=\beta\\
}} \frac{\left(\langle\xi\rangle^{\frac{1}{2}}\langle x\rangle^{-\frac{1}{2}}\right)^{|\alpha|}}{\alpha ! \beta_{1} ! \beta_{2} ! \beta_{3} !} \\
& \times \sum_{\substack{|\gamma| \leq|\beta| \\
\gamma_{1} \leq \gamma|\leq| \leq\left|\beta_{1}\right|}} \psi_{\beta \gamma \gamma_{1}}(\xi) \phi_{\beta_{1} \delta \gamma \gamma_{1}}(x) \sum_{\left|\delta^{\prime}\right| \leq\left|\beta_{2}\right|} \phi_{\beta_{2} \delta^{\prime} 00}(x) \\
& \times \int_{Q} \sigma^{\alpha+\gamma_{1}+\delta_{1}+\delta_{1}^{\prime}} \partial^{\gamma+\delta} q(\sigma) \partial^{\delta^{\prime}} q(\sigma) d \sigma \cdot \partial_{\tilde{\zeta}}^{\alpha} D_{x}^{\beta_{3}} p(x, \xi)
\end{aligned}
$$

belongs to $\mathbf{S G}^{\left(m_{1}-\frac{1}{2}(|\alpha|+|\beta|), m_{2}-\frac{1}{2}(|\beta|+|\alpha|)\right)}$. Hence,

$$
\sum_{|\alpha+\beta|=j} q_{\alpha, \beta} \in \mathbf{S G}^{m-\frac{1}{2}(j, j)}
$$

Then, we can find a symbol $t(x, \xi)$ such that

$$
t(x, \xi) \sim \sum_{j \in \mathbb{N}_{0}} \sum_{|\alpha+\beta|=j} q_{\alpha, \beta}(x, \xi) .
$$

Since, for every $N \in \mathbb{N}$,

$$
\tilde{p}_{\beta}(x, \xi)-\sum_{|\alpha|<N} q_{\alpha, \beta}(x, \xi) \in \mathbf{S G}_{\left(\frac{1}{2}, 1\right),\left(0, \frac{1}{2}\right)}^{m-\frac{1}{2}(N+|\beta|, N+|\beta|)}\left(\mathbb{R}^{2 n}\right),
$$

we obtain that $p_{F, L}-t \in \mathscr{S}\left(\mathbb{R}^{2 n}\right)$, and therefore

$$
p_{F, L}(x, \xi) \sim \sum_{j \in \mathbb{N}_{0}} \sum_{|\alpha+\beta|=j} q_{\alpha, \beta}(x, \xi)
$$

To finish the proof, let us analyze more carefully the functions $q_{\alpha, \beta}(x, \xi)$ when $|\alpha+\beta| \leq 1$. First, we notice that if $\alpha=\beta=0$, we have $q_{0,0}(x, \xi)=p(x, \xi)$. If $|\alpha|=1$ and $\beta=0$,

$$
q_{\alpha, 0}(x, \xi)=\frac{\langle\xi\rangle^{\frac{|\alpha|}{2}}\langle x\rangle^{-\frac{|\alpha|}{2}}}{\alpha !} \int \sigma^{\alpha} q(\sigma)^{2} d \sigma=0
$$


because $\sigma^{\alpha} q^{2}(\sigma)$ is an odd function. In the case $|\alpha|=0$ and $|\beta|=1$, we have

$$
\begin{aligned}
q_{0, \beta}(x, \xi) & =\sum_{\beta_{1}+\beta_{2}+\beta_{3}=\beta} D_{x}^{\beta_{3}} p(x, \xi) \sum_{\substack{|\gamma| \leq|\beta| \\
\left|\gamma_{1}\right| \leq|\gamma|}} \sum_{|\delta| \leq\left|\beta_{1}\right|} \psi_{\beta \gamma \gamma_{1}}(\xi) \phi_{\beta_{1} \delta \gamma \gamma_{1}}(x) \\
& \times \sum_{\left|\delta^{\prime}\right| \leq\left|\beta_{2}\right|} \phi_{\beta_{2} \delta^{\prime} 00}(x) \int \sigma^{\gamma_{1}+\delta+\delta^{\prime}}\left(\partial^{\gamma+\delta} q\right)(\sigma)\left(\partial^{\delta^{\prime}} q\right)(\sigma) d \sigma .
\end{aligned}
$$

If $\left|\gamma_{1}\right|<|\gamma|$ in the above formula, we have $\gamma_{1}=0$ and $|\gamma|=1$, and, since $q$ is even,

$$
\int \sigma^{\delta+\delta^{\prime}}\left(\partial^{e_{j}+\delta} q\right)(\sigma)\left(\partial^{\delta^{\prime}} q\right)(\sigma) d \sigma=0, j=1, \ldots, n .
$$

Therefore,

$$
\begin{aligned}
q_{0, \beta}(x, \xi) & =\sum_{\beta_{1}+\beta_{2}+\beta_{3}=\beta} D_{x}^{\beta_{3}} p(x, \xi) \sum_{|\gamma| \leq|\beta||\delta| \leq\left|\beta_{1}\right|} \psi_{\beta \gamma \gamma}(\xi) \phi_{\beta_{1} \delta \gamma \gamma}(x) \\
& \times \sum_{\left|\delta^{\prime}\right| \leq\left|\beta_{2}\right|} \phi_{\beta_{2} \delta^{\prime} 00}(x) \int \sigma^{\gamma+\delta+\delta^{\prime}}\left(\partial^{\gamma+\delta} q\right)(\sigma)\left(\partial^{\delta^{\prime}} q\right)(\sigma) d \sigma,
\end{aligned}
$$

and by Lemma $3 q_{0, \beta} \in \mathbf{S G}^{m-(1,1)}\left(\mathbb{R}^{2 n}\right)$. Hence

$$
p_{F, L}(x, \xi)-p(x, \xi) \sim \sum_{|\beta|=1} q_{0, \beta}(x, \xi)+\sum_{|\alpha+\beta| \geq 2} q_{\alpha, \beta}(x, \xi)
$$

and in particular that $p_{F, L}-p \in \mathbf{S G}^{m-(1,1)}\left(\mathbb{R}^{2 n}\right)$.

Proposition 6 and Theorem 4 imply the well known sharp Gårding inequality.

Theorem 5. Let $p \in S G^{m}\left(\mathbb{R}^{2 n}\right)$. If Re $p(x, \xi) \geq 0$, then

$$
\operatorname{Re}(p(x, D) u, u)_{L^{2}} \geq-C\|u\|_{H^{\frac{1}{2}(m-(1,1))}}^{2} \quad u \in \mathscr{S}\left(\mathbb{R}^{n}\right),
$$

for some positive constant $C$.

Proof. Setting $q=p-p_{F, L} \in \mathbf{S G}^{m-(1,1)}\left(\mathbb{R}^{2 n}\right)$ and recalling that $p_{F}$ and $p_{F, L}$ define the same operator, we may write, by (iv) of Proposition 6:

$$
\operatorname{Re}(p(x, D) u, u)_{L^{2}}=\operatorname{Re}(q(x, D) u, u)_{L^{2}}+\operatorname{Re}\left(p_{F} u, u\right)_{L^{2}} \geq \operatorname{Re}(q(x, D) u, u)_{L^{2}} .
$$

Now, observe that for any $s=\left(s_{1}, s_{2}\right) \in \mathbb{R}^{2}$

$$
\begin{aligned}
\left|(q(x, D) u, u)_{L^{2}}\right| & =\left|\left(\langle x\rangle^{s_{2}}\left\langle D_{x}\right\rangle^{s_{1}} q(x, D) u,\langle x\rangle^{-s_{2}}\left\langle D_{x}\right\rangle^{-s_{1}} u\right)_{L^{2}}\right| \\
& \leq\|q(x, D) u\|_{H^{s}}\|u\|_{H^{-s}} \leq C\|u\|_{H^{s+m-(1,1)}}\|u\|_{H^{-s}}
\end{aligned}
$$

Choosing $s=\frac{1}{2}[(1,1)-m]$, we conclude that

$$
\operatorname{Re}(p(x, D) u, u)_{L^{2}} \geq-C\|u\|_{H^{\frac{1}{2}(m-(1,1))}}^{2} .
$$


To study the Friedrichs part of symbols satisfying Gevrey estimates, we need the Faà di Bruno formula. Given smooth functions $g: \mathbb{R}^{n} \rightarrow \mathbb{R}^{p}, g=\left(g_{1}, \ldots, g_{p}\right), f: \mathbb{R}^{p} \rightarrow \mathbb{R}$ and $\gamma \in \mathbb{N}_{0}^{n}-\{0\}$, we have

$$
\partial^{\gamma}(f \circ g)(x)=\sum \frac{\gamma !}{k_{1} ! \ldots k_{\ell} !}\left(\partial^{k_{1}+\cdots+k_{n}} f\right)(g(x)) \prod_{j=1}^{\ell} \prod_{i=1}^{p}\left[\frac{1}{\delta_{j} !} \partial^{\delta_{j}} g_{i}(x)\right]^{k_{j i}},
$$

where the sum is taken over all $\ell \in \mathbb{N}$, all sets $\left\{\delta_{1}, \ldots, \delta_{\ell}\right\}$ of $\ell$ distinct elements of $\mathbb{N}_{0}^{n}-\{0\}$ and all $\left(k_{1}, \ldots, k_{\ell}\right) \in\left(\mathbb{N}_{0}^{p}-\{0\}\right)^{\ell}$, such that

$$
\gamma=\sum_{s=1}^{\ell}\left|k_{s}\right| \delta_{s}
$$

It is possible to show that there is a constant $C>0$ such that

$$
\sum \frac{\left(k_{1}+\ldots+k_{\ell}\right) !}{k_{1} ! \ldots k_{\ell} !} \leq C^{|\gamma|+1}, \quad \gamma \in \mathbb{N}-\{0\},
$$

and $\left|k_{1}+\ldots+k_{\ell}\right| ! \leq|\gamma| !$, where the summation and $k_{1}, \ldots, k_{\ell}$ are as in (17). For a proof of these assertions, we refer to Proposition 4.3 (Page 9), Corollary 4.5 (Page 11) and Lemma 4.8 (Page 12) of [24].

Let $p \in \mathbf{S G}_{(\mu, v)}^{m}$. We already know that $p_{F} \in \mathbf{S G}_{\left(\frac{1}{2}, 1\right),\left(0, \frac{1}{2}\right)}^{(0,0),\left(m_{1}\right)}$. Now, we want to obtain a precise information about the Gevrey regularity of $p_{F}$. By Faà di Bruno formula,

$$
\begin{aligned}
& \partial_{x}^{\beta} \partial_{\xi}^{\alpha} q\left(\langle\xi\rangle^{-\frac{1}{2}}\langle x\rangle^{\frac{1}{2}}(\zeta-\xi)\right)=\partial_{x}^{\beta} \sum_{\ell, k_{1}, \ldots, k_{\ell}} \frac{\alpha !}{k_{1} ! \ldots k_{\ell} !} \\
& \quad \times\left(\partial^{k_{1}+\ldots+k_{\ell}} q\right)\left(\langle\xi\rangle^{-\frac{1}{2}}\langle x\rangle^{\frac{1}{2}}(\zeta-\xi)\right) \prod_{j=1}^{\ell}\langle x\rangle^{\frac{\left|k_{j}\right|}{2}} \prod_{i=1}^{n}\left[\frac{1}{\delta_{j} !} \partial_{\xi}^{\delta_{j}}\left\{\langle\xi\rangle^{-\frac{1}{2}}\left(\zeta_{i}-\xi_{i}\right)\right\}\right]^{k_{j i}} \\
& \quad=\sum_{\ell, k_{1}, \ldots, k_{\ell}} \frac{\alpha !}{k_{1} ! \ldots k_{\ell} !} \sum_{\beta_{1}+\beta_{2}=\beta} \frac{\beta !}{\beta_{1} ! \beta_{2} !} \partial_{x}^{\beta_{1}}\left(\partial^{k_{1}+\ldots+k_{\ell}} q\right)\left(\langle\xi\rangle^{-\frac{1}{2}}\langle x\rangle^{\frac{1}{2}}(\zeta-\xi)\right) \\
& \quad \times \partial_{x}^{\beta_{2}} \prod_{j=1}^{\ell}\langle x\rangle^{\frac{\left|k_{j}\right|}{2}} \prod_{i=1}^{n}\left[\frac{1}{\delta_{j} !} \partial_{\xi}^{\delta_{j}}\left\{\langle\xi\rangle^{-\frac{1}{2}}\left(\zeta_{i}-\xi_{i}\right)\right\}\right]^{k_{j i}} \\
& \quad=\sum_{\ell, k_{1}, \ldots, k_{\ell}} \frac{\alpha !}{k_{1} ! \ldots k_{\ell} !} \sum_{\beta_{1}+\beta_{2}=\beta} \frac{\beta !}{\beta_{1} ! \beta_{2} !} \sum_{\ell^{\prime}, k_{1^{\prime}, \ldots, k_{\ell^{\prime}}} \frac{\beta_{1} ! \ldots k_{\ell^{\prime}} !}{k_{1}^{\prime} !}} \times\left(\partial^{\left(k_{1}+\ldots+k_{\ell}\right)+\left(k_{1}^{\prime}+\ldots+k_{\ell^{\prime}}^{\prime}\right.} q\right)\left(\langle\xi\rangle^{-\frac{1}{2}}\langle x\rangle^{\frac{1}{2}}(\zeta-\xi)\right) \\
& \quad \times \prod_{j^{\prime}=1}^{\ell^{\prime}} \prod_{i^{\prime}=1}^{n}\left[\frac{1}{\delta_{j^{\prime}}^{\prime} !} \partial_{x}^{\delta_{j}^{\prime}}\langle\xi\rangle^{-\frac{1}{2}}\langle x\rangle^{\frac{1}{2}}\left(\zeta_{i^{\prime}}-\xi_{i^{\prime}}\right)\right]^{k_{j^{\prime} i^{\prime}}^{\prime}} \\
& \quad \times \sum_{\sigma_{1}+\ldots+\sigma_{\ell}=\beta_{2}} \frac{\beta_{2} !}{\sigma_{1} ! \ldots \sigma_{\ell} !} \prod_{j=1}^{\ell} \partial_{x}^{\sigma_{j}}\langle x\rangle^{\frac{\left|k_{j}\right|}{2}} \prod_{i=1}^{n}\left[\frac{1}{\delta_{j} !} \partial_{\xi}^{\delta_{j}}\left\{\langle\xi\rangle^{-\frac{1}{2}}\left(\zeta_{i}-\xi_{i}\right)\right\}\right]^{k_{j i}},
\end{aligned}
$$


Mathematics 2020, 8, 1938

20 of 23

hence

$$
\begin{aligned}
& \partial_{x}^{\beta} \partial_{\xi}^{\alpha} q\left(\langle\xi\rangle^{-\frac{1}{2}}\langle x\rangle^{\frac{1}{2}}(\zeta-\xi)\right)=\sum_{\ell, k_{1}, \ldots, k_{\ell}} \frac{\alpha !}{k_{1} ! \ldots k_{\ell} !} \sum_{\beta_{1}+\beta_{2}=\beta} \frac{\beta !}{\beta_{1} ! \beta_{2} !} \\
& \quad \times \sum_{\ell^{\prime}, k_{1}^{\prime}, \ldots, k_{\ell^{\prime}}^{\prime}} \frac{\beta_{1} !}{k_{1}^{\prime} ! \ldots k_{\ell^{\prime}}^{\prime} !}\left(\partial^{\left(k_{1}+\ldots+k_{\ell}\right)+\left(k_{1}^{\prime}+\ldots+k_{\ell^{\prime}}^{\prime}\right)} q\right)\left(\langle\xi\rangle^{-\frac{1}{2}}\langle x\rangle^{\frac{1}{2}}(\zeta-\xi)\right) \\
& \quad \times \prod_{j^{\prime}=1}^{\ell^{\prime}} \prod_{i^{\prime}=1}^{n}\left[\frac{1}{\delta_{j^{\prime}} !} \partial_{x}^{\delta_{j^{\prime}}^{\prime}}\langle x\rangle^{\frac{1}{2}}\langle x\rangle^{-\frac{1}{2}}\right]^{k_{j^{\prime} i^{\prime}}^{\prime}}\left[\langle\xi\rangle^{-\frac{1}{2}}\langle x\rangle^{\frac{1}{2}}\left(\zeta_{i^{\prime}}-\xi_{i^{\prime}}\right)\right]^{k_{j^{\prime} i^{\prime}}^{\prime}} \\
& \quad \times \sum_{\sigma_{1}+\ldots+\sigma_{\ell}=\beta_{2}} \frac{\beta_{2} !}{\sigma_{1} ! \ldots \sigma_{\ell} !} \prod_{j=1}^{\ell} \partial_{x}^{\sigma_{j}}\langle x\rangle^{\frac{1}{2}\left|k_{j}\right|} \prod_{i=1}^{n}\left[\frac{1}{\delta_{j} !}\left\{\partial_{\xi}^{\delta_{j}}\langle\xi\rangle^{-\frac{1}{2}}\left(\zeta_{i}-\xi_{i}\right)-\delta_{j i} \partial_{\xi}^{\delta_{j}-e_{i}}\langle\xi\rangle^{-\frac{1}{2}}\right\}\right]^{k_{j i}} .
\end{aligned}
$$

Noticing that $\langle x\rangle^{\frac{1}{2}}\langle\xi\rangle^{-\frac{1}{2}}|\zeta-\xi| \leq 1$ on the support of $q$, we have

$$
\left|\partial_{x}^{\beta} \partial_{\xi}^{\alpha} q\left(\langle\xi\rangle^{-\frac{1}{2}}\langle x\rangle^{\frac{1}{2}}(\zeta-\xi)\right)\right| \leq \tilde{C}_{q, S}^{|\alpha+\beta|+1}(\alpha ! \beta !)^{\mathcal{S}}\langle x\rangle^{\frac{|\alpha|}{2}-|\beta|}\langle\xi\rangle^{-\frac{|\alpha|}{2}} .
$$

We now apply the above inequality to estimate the derivatives of $F$. We have

$$
\begin{aligned}
&\left|\partial_{\xi}^{\alpha} \partial_{x}^{\beta} F(x, \xi, \zeta)\right| \leq \sum_{\substack{\alpha_{1}+\alpha_{2}=\alpha \\
\beta_{1}+\beta_{1}=\beta}} \frac{\alpha ! \beta !}{\alpha_{1} ! \beta_{1} ! \alpha_{2} ! \beta_{2} !} \partial_{\xi}^{\alpha_{1}}\langle\xi\rangle^{-\frac{n}{4}} \partial_{x}^{\beta_{1}}\langle x\rangle^{\frac{n}{4}}\left|\partial_{\xi}^{\alpha_{2}} \partial_{x}^{\beta_{2}} q\left(\langle\xi\rangle^{-\frac{1}{2}}\langle x\rangle^{\frac{1}{2}}(\zeta-\xi)\right)\right| \\
& \leq C_{q, s}^{|\alpha+\beta|+1}(\alpha ! \beta !)^{\mathcal{S}}\langle\xi\rangle^{-\frac{n}{4}-\frac{|\alpha|}{2}}\langle x\rangle^{\frac{n}{4}+\frac{|\alpha|}{2}-|\beta|} .
\end{aligned}
$$

Finally we proceed with the estimates for $p_{F}$. Denoting

$$
Q_{x, \xi}=\left\{\zeta \in \mathbb{R}^{n}:\langle x\rangle^{\frac{1}{2}}\langle\xi\rangle^{-\frac{1}{2}}|\zeta-\xi|<1\right\}, \quad x, \xi \in \mathbb{R}^{n}
$$

we obtain

$$
\begin{aligned}
& \left|\partial_{\xi}^{\alpha} \partial_{\xi^{\prime}}^{\alpha^{\prime}} \partial_{x^{\prime}}^{\beta^{\prime}} p_{F}\left(\xi, x^{\prime}, \xi^{\prime}\right)\right| \leq \sum_{\beta_{1}+\beta_{2}+\beta_{2}=\beta} \frac{\beta !}{\beta_{1} ! \beta_{2} ! \beta_{3} !}\left[\int_{Q_{x, \xi}}\left|\partial_{\xi}^{\alpha} \partial_{x}^{\beta_{1}^{\prime}} F\left(\xi, x^{\prime}, \zeta\right)\right|^{2} d \zeta\right]^{\frac{1}{2}} \\
& \times\left[\int_{Q_{x^{\prime}, \xi^{\prime}}}\left|\partial_{x}^{\beta_{3}^{\prime}} p\left(x^{\prime}, \zeta\right) \partial_{\xi}^{\alpha^{\prime}} \partial_{x}^{\beta_{2}^{\prime}} F\left(x^{\prime}, \xi^{\prime}, \zeta\right)\right|^{2} d \zeta\right]^{\frac{1}{2}} \\
& \leq \sum_{\beta_{1}+\beta_{2}+\beta_{2}=\beta} \frac{\beta !}{\beta_{1} ! \beta_{2} ! \beta_{3} !} C_{q, s}^{\left|\alpha+\alpha^{\prime}+\beta_{1}^{\prime}+\beta_{2}^{\prime}\right|+2}\left(\alpha ! \alpha^{\prime} ! \beta_{1}^{\prime} ! \beta_{2}^{\prime} !\right)^{S}\langle\xi\rangle^{-\frac{|\alpha|}{2}}\left\langle\xi^{\prime}\right\rangle^{-\frac{\left|\alpha^{\prime}\right|}{2}} \\
& \times\langle x\rangle^{\frac{1}{2}\left|\alpha+\alpha^{\prime}\right|-\left|\beta_{1}^{\prime}+\beta_{2}^{\prime}\right|}\left[\int_{Q_{x^{\prime}, \xi}}\langle\xi\rangle^{-\frac{n}{2}}\left\langle x^{\prime}\right\rangle^{\frac{n}{2}} d \zeta\right]^{\frac{1}{2}} \cdot\left[\int_{Q_{x^{\prime}, \xi^{\prime}}}\left\langle\xi^{\prime}\right\rangle^{-\frac{n}{2}}\left\langle x^{\prime}\right\rangle^{\frac{n}{2}}\left|\partial_{x}^{\beta_{3}^{\prime}} p\left(x^{\prime}, \zeta\right)\right|^{2} d \zeta\right]^{\frac{1}{2}} \\
& \leq \sum_{\beta_{1}+\beta_{2}+\beta_{2}=\beta} \frac{\beta !}{\beta_{1} ! \beta_{2} ! \beta_{3} !} C_{q, s}^{\left|\alpha+\alpha^{\prime}+\beta_{1}^{\prime}+\beta_{2}^{\prime}\right|+2}\left(\alpha ! \alpha^{\prime} ! \beta_{1}^{\prime} ! \beta_{2}^{\prime} !\right)^{s}\langle\xi\rangle^{-\frac{|\alpha|}{2}}\left\langle\xi^{\prime}\right\rangle^{-\frac{\left|\alpha^{\prime}\right|}{2}}
\end{aligned}
$$

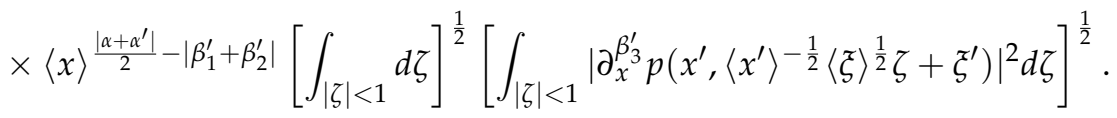

Using Lemma 4 and recalling that $s \leq \min \{\mu, v\}$,

$$
\left|\partial_{\xi}^{\alpha} \partial_{\xi^{\prime}}^{\alpha^{\prime}} \partial_{x^{\prime}}^{\beta^{\prime}} p_{F}\right| \leq C^{\left|\alpha+\alpha^{\prime}+\beta^{\prime}\right|+1}\left(\alpha ! \alpha^{\prime} !\right)^{\mu} \beta^{\prime} !^{v}\langle x\rangle^{m_{2}-\left|\beta^{\prime}\right|+\frac{1}{2}\left|\alpha+\alpha^{\prime}\right|}\langle\xi\rangle^{-\frac{|\alpha|}{2}}\left\langle\xi^{\prime}\right\rangle^{m_{1}-\frac{\left|\alpha^{\prime}\right|}{2}},
$$

which means $p_{F} \in \mathbf{S G}_{\left(\frac{1}{2}, 1\right),\left(0, \frac{1}{2}\right) ;(\mu, v)}^{(0,0),\left(m_{1}, m_{2}\right)}\left(\mathbb{R}^{4 n}\right)$. 
Now, we discuss the asymptotic expansion of $p_{F}$, when $p \in \mathbf{S G}_{(\mu, v)}^{m}$. In the following, we use the notation of the proof of Theorem 4 . We have

$$
p_{F, L}(x, \xi) \sim \sum_{\beta} \tilde{p}_{\beta}(x, \xi) \text { in } \operatorname{FSG}_{\left(\frac{1}{2}, 1\right),\left(0, \frac{1}{2}\right) ;(\tilde{\mu}, \tilde{v})^{\prime}}^{m}
$$

and, by Lemma 2 and Taylor formula, we may write

$$
\left|\partial_{\tilde{\xi}}^{\theta} \partial_{x}^{\sigma}\left(p_{F, L}-\sum_{|\beta|<N} \tilde{p}_{\beta}\right)(x, \xi)\right| \leq C^{|\theta+\sigma|+2 N+1} \theta ! \tilde{\mu} \sigma !^{\tilde{v}} N !^{\tilde{\mu}+\tilde{v}-1}\langle\xi\rangle^{m_{1}-\frac{|\theta|}{2}-\frac{N}{2}}\langle x\rangle^{m_{2}-|\sigma|+\frac{|\theta|}{2}-\frac{N}{2}}
$$

for every $\theta, \sigma \in \mathbb{N}_{0}^{n}, x, \xi \in \mathbb{R}^{n}$ and $N \in \mathbb{N}$, where $\tilde{\mu}=3 \mu+\frac{1}{2} v$ and $\tilde{v}=v+2 \mu$. We also have, for every $\beta \in \mathbb{N}_{0}^{n}$ and $N \in \mathbb{N}$,

$$
\tilde{p}_{\beta}(x, \xi)-\sum_{|\alpha|<N} q_{\alpha, \beta}(x, \xi)=r_{\beta, N}(x, \xi) .
$$

where $r_{\beta, N}$ is given as in (16).

Changing variables and setting $\sigma=(\zeta-\xi)\langle x\rangle^{\frac{1}{2}}\langle\xi\rangle^{-\frac{1}{2}}$, we obtain

$$
\begin{aligned}
& r_{\beta, N}(x, \xi)=\sum_{\beta_{1}+\beta_{2}+\beta_{3}=\beta|\alpha|=N} \sum_{\alpha !} \frac{N}{\alpha !} \cdot \frac{\left(\langle\xi\rangle^{\frac{1}{2}}\langle x\rangle^{-\frac{1}{2}}\right)^{N}}{\beta_{1} ! \beta_{2} ! \beta_{3} !} \\
& \times \sum_{\substack{|\gamma| \leq|\beta| \\
\gamma_{1} \leq \gamma|\leq| \leq\left|\beta_{1}\right|}} \psi_{\beta \gamma \gamma_{1}}(\xi) \phi_{\beta_{1} \delta \gamma \gamma_{1}}(x) \sum_{\left|\delta^{\prime}\right| \leq\left|\beta_{2}\right|} \phi_{\beta_{2} \delta^{\prime} 00}(x) \\
& \times \int_{Q_{x, \xi}}\left((\zeta-\xi)\langle x\rangle^{\frac{1}{2}}\langle\xi\rangle^{-\frac{1}{2}}\right)^{\gamma_{1}+\delta+\delta^{\prime}+\alpha} \partial^{\gamma+\delta} q\left((\zeta-\xi)\langle x\rangle^{\frac{1}{2}}\langle\xi\rangle^{-\frac{1}{2}}\right) \partial^{\delta^{\prime}} q\left((\zeta-\xi)\langle x\rangle^{\frac{1}{2}}\langle\xi\rangle^{-\frac{1}{2}}\right) \\
& \cdot \int_{0}^{1}(1-\theta)^{N-1}\left(\partial_{\xi}^{\alpha} D_{x}^{\beta_{3}} p\right)(x, \theta \zeta+(1-\theta) \xi) d \theta\langle x\rangle^{\frac{n}{2}}\langle\xi\rangle^{-\frac{n}{2}} d \zeta .
\end{aligned}
$$

By Lemma 3, we get

$$
\begin{aligned}
r_{\beta, N}(x, \xi) & =\sum_{\beta_{1}+\beta_{2}+\beta_{3}=\beta|\alpha|=N} \frac{N}{\alpha !} \cdot \frac{\left(\langle\xi\rangle^{\frac{1}{2}}\langle x\rangle^{-\frac{1}{2}}\right)^{N}}{\beta_{1} ! \beta_{2} ! \beta_{3} !} \\
& \times \int_{Q_{x, \xi}}\left(\partial_{\xi}^{\beta} \partial_{x}^{\beta_{1}} F\right)(x, \xi, \zeta)\left(\partial_{x}^{\beta_{2}} F\right)(x, \xi, \zeta) \int_{0}^{1}(1-\theta)^{N-1}\left(\partial_{\xi}^{\alpha} D_{x}^{\beta_{3}} p\right)(x, \theta \zeta+(1-\theta) \xi) d \theta d \zeta .
\end{aligned}
$$

Now, there exists $K>0$ such that

$$
K^{-1}\langle\xi\rangle \leq\langle\theta \zeta+(1-\theta) \xi\rangle \leq K\langle\xi\rangle, \quad|\theta|<1, \zeta \in Q_{x, \xi}, x, \xi \in \mathbb{R}^{n} .
$$

Then, using (18), since $s \leq \min \{\mu, v\}$, we obtain

$$
\begin{aligned}
& \left|\partial_{\xi}^{\gamma} \partial_{x}^{\delta} r_{\beta, N}(x, \xi)\right| \leq C^{|\gamma+\delta|+2(N+|\beta|)+1} \gamma !^{\mu} \delta !^{v} \beta !^{s+v-1} N !^{\mu-1} \\
& \times\langle\xi\rangle^{m_{1}-|\gamma|-\frac{N+|\beta|}{2}}\langle x\rangle^{m_{2}-|\delta|-\frac{N+|\beta|}{2}} \underbrace{\int_{Q_{x, \xi}}\langle\xi\rangle^{-\frac{n}{2}}\langle x\rangle^{\frac{n}{2}} d \sigma}_{=\int_{|\sigma| \leq 1} d \sigma} \\
& \leq C^{|\gamma+\delta|+2(N+|\beta|)+1} \gamma !^{\mu} \delta !^{v} \beta !^{s+v-1} N !^{\mu-1}\langle\xi\rangle^{m_{1}-|\gamma|-\frac{N+|\beta|}{2}}\langle x\rangle^{m_{2}-|\delta|-\frac{N+|\beta|}{2}},
\end{aligned}
$$

for every $\gamma, \delta \in \mathbb{N}_{0}^{n}, x, \xi \in \mathbb{R}^{n}$ and $N \in \mathbb{N}$. Now, by (19) and (20), we get

$$
p_{F, L}(x, \xi) \sim \sum_{j \in \mathbb{N}_{0}} \sum_{|\alpha+\beta|=j} q_{\alpha, \beta}(x, \xi) \text { in } F_{\left(\frac{1}{2}, 1\right),\left(0, \frac{1}{2}\right) ;(\tilde{\mu}, \tilde{v})}^{m} .
$$


To improve the above asymptotic expansion, note that, for $j \geq 2$,

$$
\left|\partial_{\xi}^{\gamma} \partial_{x}^{\delta} \sum_{|\alpha+\beta|=j} q_{\alpha, \beta}(x, \xi)\right| \leq C^{|\gamma+\delta|+2 j+1} \gamma !^{\mu} \delta !^{v} j !^{\mu+v-1}\langle x\rangle^{m_{2}-|\delta|-\frac{j}{2}}\langle\xi\rangle^{m_{1}-|\gamma|-\frac{j}{2}},
$$

and

$$
\left|\partial_{\xi}^{\gamma} \partial_{x}^{\delta} \sum_{|\beta|=1} q_{0, \beta}(x, \xi)\right| \leq C^{|\theta+\sigma|+2 j+1} \gamma !^{\mu} \delta !^{v} j !^{\mu+v-1}\langle x\rangle^{m_{2}-|\delta|-1}\langle\xi\rangle^{m_{1}-|\gamma|-1},
$$

for every $\gamma, \delta \in \mathbb{N}_{0}^{n}, x, \xi \in \mathbb{R}^{n}$, hence

$$
\sum_{j \in \mathbb{N}_{0}} \sum_{|\alpha+\beta|=j} q_{\alpha, \beta}(x, \xi) \in F_{\left(k_{j}, \ell_{j}\right)} S G_{(\mu, v)}^{m}
$$

where $k_{0}=\ell_{0}=0, k_{1}=\ell_{1}=1, k_{j}=\ell_{j}=\frac{j}{2}$. Then, there exists $q \in \mathbf{S G}_{(\mu, v)}^{m}\left(\mathbb{R}^{2 n}\right)$ such that

$$
q(x, \xi) \sim \sum_{\alpha, \beta} q_{\alpha, \beta}(x, \xi) \text { in } F_{\left(k_{j}, \ell_{j}\right)} S G_{(\mu, v)}^{m} .
$$

Repeating the argument at the end of Section 3, we can write $p_{F, L}(x, \xi)=q(x, \xi)+r(x, \xi)$, where $r$ belongs to the Gelfand-Shilov space $\mathcal{S}_{\tilde{\mu}+\tilde{v}-1}\left(\mathbb{R}^{2 n}\right)$. Summing up, we obtain the following result.

Theorem 6. Let $p \in S G_{(\mu, v)}^{m}$ and $p_{F}$ be its Friedrichs part. Then, we can write $p_{F, L}=q+r$, with $r \in \mathcal{S}_{\tilde{\mu}+\tilde{v}-1}\left(\mathbb{R}^{2 n}\right)$ and

$$
q(x, \xi) \sim p(x, \xi)+\sum_{|\beta|=1} q_{0, \beta}(x, \xi)+\sum_{|\alpha+\beta| \geq 2} q_{\alpha, \beta}(x, \xi) \text { in } F_{\left(k_{j}, \ell_{j}\right)} S G_{(\mu, v)}^{m}
$$

where $k_{0}=\ell_{0}=0, k_{1}=\ell_{1}=1, k_{j}=\ell_{j}=\frac{j}{2}$. Moreover, the symbols $q_{0, \beta} \in S G_{(\mu, v)}^{m-(1,1)}\left(\mathbb{R}^{2 n}\right)$ and $q_{\alpha, \beta} \in S G_{(\mu, v)}^{m-\frac{|\alpha+\beta|}{2}(1,1)}\left(\mathbb{R}^{2 n}\right)$ are the same as in Theorem 4 .

Author Contributions: Conceptualization, A.A.J. and M.C.; methodology, A.A.J. and M.C.; formal analysis, A.A.J. and M.C.; investigation, A.A.J. and M.C.; writing-original draft preparation, A.A.J. and M.C.; writing-review and editing, A.A.J. and M.C. All authors have read and agreed to the published version of the manuscript.

Funding: This research received no external funding.

Acknowledgments: The first author would like to thank Fundação Araucária for the financial support during the development of this paper.

Conflicts of Interest: The authors declare no conflict of interest.

\section{References}

1. Hörmander, L. Pseudo-differential operators and non-elliptic noundary problems. Ann. Math. 1966, 83, 129-209. [CrossRef]

2. Lax, P.D.; Nirenberg, L. On stability for difference scheme: A sharp form of Gårding inequality. Commun. Pure Appl. Math. 1966, 19, 473-492. [CrossRef]

3. Friedrichs, K.O. Pseudo-Differential Operators; Lecture Note; Courant Inst. Math. Sci. New York Univ.: New York, NY, USA, 1968.

4. Hörmander, L. The Analysis of Linear Partial Differential Operators III; Springer: Berlin/Heidelberg, Germany, 1985.

5. Kumano-Go, H. Pseudo-Differential Operators; The MIT Press: Cambridge, UK; London, UK, 1982.

6. Nagase, M. A new proof of sharp Gårding inequality. Funkc. Ekvacioj 1977, 20, 259-271.

7. Cicognani, M.; Colombini, F. The Cauchy problem for $p$-evolution equations. Trans. Am. Math. Soc. 2010, 362, 4853-4869. [CrossRef] 
8. Cicognani, M.; Reissig, M. Well-posedness for degenerate Schrödinger equations. Evol. Equ. Control Theory 2014, 3, 15-33. [CrossRef]

9. Ascanelli, A.; Boiti, C.; Zanghirati, L. Well-posedness of the Cauchy problem for p-evolution equations. J. Differ. Equ. 2012, 253 2765-2795. [CrossRef]

10. Ascanelli, A.; Cappiello, M. Weighted energy estimates for p-evolution equations in SGclasses. J. Evol. Equ. 2015, 15, 583-607. [CrossRef]

11. Gelfand, I.M.; Shilov, G.E. Generalized Functions; Academic Press: New York, NY, USA; London, UK, 1967; Volume 2.

12. Ascanelli, A.; Cappiello, M. Schrödinger-type equations in Gefand-Shilov spaces. J. Math. Pures Appl. 2019, 132, 207-250. [CrossRef]

13. Arias, A., Jr.; Ascanelli, A.; Cappiello, M. The Cauchy problem for 3-evolutions equations with data in Gelfand-Shilov spaces. arXiv 2020, arXiv:2009.10366.

14. Cappiello, M.; Rodino, L. SG-pseudodifferential operators and Gelfand-Shilov spaces. Rocky Mt. J. Math. 2006, 36, 1118-1148. [CrossRef]

15. Cordes, H.O. The Technique of Pseudo-Differential Operators; Cambridge Univ. Press: Cambridge, UK, 1995.

16. Coriasco, S. Fourier integral operators in SG classes.I. Composition theorems and action on SG-Sobolev spaces. Rend. Sem. Mat. Univ. Pol. Torino 1999, 57, 249-302.

17. Coriasco, S. Fourier integral operators in SG classes.II. Application to SG hyperbolic Cauchy problems. Ann. Univ. Ferrara Sez VII 1998, 44, 81-122.

18. Egorov, Y.V.; Schulze, B.-W. Pseudo-Differential Operators, Singularities, Applications; Operator Theory: Advances and Applications 93; Birkhäuser Verlag: Basel, Switzerland, 1997.

19. Nicola, F.; Rodino, L. Global Pseudo-Differential Calculus on Euclidean Spaces; Birkhäuser: Basel, Switzerland, 2010; Volume 4.

20. Parenti, C. Operatori pseudodifferenziali in $\mathbb{R}^{n}$ e applicazioni. Ann. Mat. Pura Appl. 1972, 93, 359-389. [CrossRef]

21. Schrohe, E. Spaces of weighted symbols and weighted Sobolev spaces on manifolds. In Proceedings, Oberwolfach, 1256; Cordes, H.O., Gramsch, B., Widom, H., Eds.; Springer: New York, NY, USA, 1986; pp. 360-377.

22. Camperi, I. Global hypoellipticity and Sobolev estimates for generalized SG-pseudo-differential operators. Rend. Sem. Mat. Univ. Pol. Torino 2008, 66, 99-112.

23. Cappiello, M.; Gramchev, T.; Rodino, L. Sub-exponential decay and uniform holomorphic extensions for semilinear pseudodifferential equations. Commun. Partial Differ. Equ. 2010, 35, 846-877. [CrossRef]

24. Bierstone, E.; Milman, P.D. Resolution of singularities in Denjoy-Carleman classes. Sel. Math. 2004, $10,1$. [CrossRef]

Publisher's Note: MDPI stays neutral with regard to jurisdictional claims in published maps and institutional affiliations.

(C) 2020 by the authors. Licensee MDPI, Basel, Switzerland. This article is an open access article distributed under the terms and conditions of the Creative Commons Attribution (CC BY) license (http:/ / creativecommons.org/licenses/by/4.0/). 